\title{
MANAGING TELEVISION COMMERCIAL INVENTORY UNDER COMPETITION: AN EQUILIBRIUM ANALYSIS
}

\author{
Qin Geng \\ College of Business, Kutztown University of Pennsylvania, Kutztown, PA 19530, geng@kutztown.edu \\ Suman Mallik \\ School of Business, University of Kansas, Lawrence, KS 66045, suman@ku.edu \\ Last Revision: March 2018 \\ Forthcoming in Decision Sciences
}

\begin{abstract}
We develop a game theoretic model for managing prime time on-air ad inventory in the television industry. The ad inventory in this industry is priced based on rating points or the number of viewers that watch a commercial. The rating points are sold through two distinct processes: the upfront, which occurs before the broadcast season, and the scatter, which occurs throughout during the broadcast season. Television networks need to allocate their total rating points inventory to these two markets before knowing either the performance rating of their shows or the scatter market price, both of which are ex ante uncertain. The television networks offer performance guarantees on the inventory that is sold in the upfront market while such guarantees are not offered in the scatter market. We consider the inventory competition between two television networks under such a setting. To the best of our knowledge, ours is the first paper to consider competition in media revenue management. We establish the existence of unique Nash equilibrium under quantity competition and describe the sensitivity of the equilibrium outcome with respect to various problem parameters. We show that choosing quantity over price during the upfront is a dominant strategy for a television network. We compare our competitive model with a centralized system and discuss the managerial implications for our work.
\end{abstract}

\section{Key Words: Inventory Management, Uncertainty, Television networks, Game Theory}

\section{INTRODUCTION}

The television networks, similar to other media outlets, derive their revenue almost entirely from advertising. Total television ad spending in the USA for the year 2016 was estimated at \$63 billion, excluding special events such as the Olympics and election spending (Flint and Vranica, 2016). As a result, managing the inventory of advertising spaces optimally is of immense importance to a television network. Yet, revenue management in the television industry has received relatively little attention in the operations management literature. There are only a handful of studies that describe revenue management in this 
industry for a single-firm (Bollapragada and Mallik, 2008; Araman and Popescu, 2010). Our objective in this paper is to develop a competitive model of managing the inventory of on-air advertising time in broadcast television industry.

The process of selling on-air advertising time (or commercial time) in the television industry exhibits many interesting features. The pricing of commercial time is based on show performances, or the actual number of people watching a show, as measured ex-post by the media rating agencies such as A.C. Nielsen. This performance measure often goes by different names, including rating points, eyeballs, and viewership. Throughout this paper, we will use term rating points to denote the number of people watching a show. As described below, commercial time could be sold based either on rating points (e.g., price per thousand rating points/impressions) or on time slots (e.g., price for a 30-second slot) at different times during a year and may or may not come with a guarantee on deliverable rating points.

The standard practice in the broadcast television industry, which has subsequently been adopted by the cable television industry as well, is to sell the inventory of commercial times through two distinct processes: the upfront selling and the scatter selling. A broadcast year in network television in the United States begins in the third week of September and runs until the following summer. The upfront typically occurs during the month of May, much before the start of the actual broadcast season. At this time, all television networks unveil their upcoming fall prime-time program schedule and tempt the big advertisers and media buyers to buy airtime in bulk for the entire broadcast year. The upfront market lasts for about two weeks. Since the show performances for the upcoming broadcast year are unknown at the time of the upfront, the television networks price sales contracts based on the guaranteed deliverable rating points using a negotiated price per rating point. An upfront contract for a customer specifies two things: (a) the guaranteed amount of rating points to be delivered at the contracted price, and (b) a minimum number of 30-second slots for the customer.

Because of the performance uncertainties of shows, if a television network is unable to deliver the guaranteed rating points in the in committed number of slots, it has to provide additional commercial slots until the agreed upon rating points are delivered. On the other hand, if the shows of a television network perform better than expected, it might be able to deliver the committed ratings points using fewer than the committed slots. Under such a scenario the television network still needs to air commercials for the customer for the committed number of slots, resulting in over delivering on the guaranteed rating points and loss of revenue. In summary, while the price of an upfront contract depends only on the deliverable rating points, both the deliverable amount of rating points and the committed number of slots are sacrosanct. The remaining inventory after the upfront is available for sale in the scatter market, which occurs throughout the broadcast season. Unlike the upfront, the commercial time is typically sold on a per-slot basis during the scatter season and that no rating point guarantee is offered. A commercial slot during a popular show, 
nevertheless, commands a higher scatter price than a slot during a less popular show. The scatter price is unknown during the upfront season as the uncertainties associated with the show performances are not resolved until the broadcast year begins. The scatter market is typically used by smaller advertisers who are unwilling or unable to make large commitments during the upfront, as well by larger advertisers who desire commercial times in excess of their upfront commitments (Phillips and Young, 2012). Selling commercial times through upfront and scatter markets has been the standard practice in the network television industry since the 1950s. Cable television industry also adopted this practice starting in the 1990s. Total upfront commitment during the 2015 -2016 broadcast year in the US was $\$ 8.36$ billion and $\$ 9.45$ billion respectively for the network television and the cable television industries (Friedman, 2015). The total upfront commitment in the network television industry is estimated at $\$ 8.75$ billion for the $2016-2017$ broadcast year (Shields, 2016).

Under the scenario described above, a television network often faces the following decision problem: how many rating points to sell during the upfront market in the presence of competing networks? We propose a game theoretic model to accomplish this. We consider two competing networks, each making a strategic decision about how to allocate the inventory of rating points between the upfront and scatter markets. Our model explicitly incorporates the performance uncertainty of the television shows as well as the price uncertainty of the scatter market. We establish unique Nash equilibrium of the Cournot game where each network chooses the amount of rating points. We show that upfront inventories of two networks are strategic substitutes and describe the sensitivity of the equilibrium outcome with respect to various problem parameters. We show that deliberately exaggerating the show performance information to its upfront customers is an optimal strategy for a television network. We find that setting quantity over price in the upfront market is a dominant strategy for each television network. We consider three extensions to our basic model to include client-level allocation of the upfront inventory, availability-and-performancedependent scatter market pricing, and information asymmetry.

While there is an extensive literature on scheduling strategies for new programs in Television, the literature on media revenue management is relatively sparse. Unlike the traditional revenue management problem where the available supply (e.g., the number of seats in an airplane or the number of rooms in a hotel) is deterministic, media revenue management problems are characterized by random supply as the show performances are unknown ex-ante. Bollapragada and Mallik (2008) and Araman and Popescu (2010) consider such problems in a single-firm setting. We make important contribution to the operations management literature by extending the media revenue management framework to a competitive setting, a first to the best of our knowledge. While there is little transparency in practice to show that the television networks engage in quantity competition, we have shown that the dominant strategy of a television network is to choose quantity (i.e., rating points) irrespective of the action of its competitor. Our work, thus, provides 
a theoretical validation for making quantity the key decision variable in media revenue management problems. Such theoretical validation is not provided by earlier works. Our work shows that the negotiated expected rating points of a show plays an important role in the upfront inventory decisions of a network. We show that a network has incentive to deliberately inflate the show performances as its expected revenue is increasing in the negotiated expected rating points. While extending our analyses to multiple client upfront planning, we show that there is a simple additive relationship of the equilibrium upfront inventories between the multiple client inventory planning and the aggregate inventory planning.

The remainder of this paper is organized as follows. The next section provides a review of the related literature. Section 3 describes our model. Our results are presented in Section 4. Section 5 describes two extensions of our basic model. We describe the managerial implications and conclude the paper in Section 6. Proofs of all of our analytical results are provided in the Appendix.

\section{LITERATURE REVIEW}

Our work is related to two streams of research: the revenue management (more specifically, the media revenue management), and the supply chain and/or marketing literature where capacity and/or inventory is sold through two-stage processes (e.g. both futures and spot markets). We provide a brief review of these two streams of literature in this section.

\subsection{Revenue Management Literature}

The vast literature on revenue management typically focuses on perishable assets such as seats in a commercial airplane or rooms in a hotel. Researchers have extensively studied both pricing and capacity management as the levers for revenue management. We refer an interested reader to the excellent reviews by Talluri and van Ryzin (2004) and Bitran and Caldentey (2003).

The key feature that distinguishes media revenue management problems from other revenue management problems is the uncertain supply. Unlike a hotel or an airline company, the amount of supply of rating points during a broadcast year (or the gross rating points GRP) is ex ante unknown to a television network and is realized only after the end of the broadcast year and/or after a show has been aired.

The literature on media revenue management looks at both scheduling of shows and inventory management of ad times as the levers of maximizing revenue. Majority of the model-based literature deals with scheduling strategies for television shows to maximize show ratings, and hence, the revenues, of a television network. Some examples of early work in this area include Goodhardt et al. (1975), Headen et al. (1979), Henry and Rinne (1984), Webster (1985), Rust and Echambadi (1989), and Reddy et al. (1998). More recently, both sales planning and scheduling of television commercials have been studied by Bollapragada et al. (2002), Bollapragada and Garbiras (2004), and Bollapragada, Bussieck, and Mallik (2004). Gaur, Krishnamurti, and Kohli (2009) consider an extension of Bollapragada, Bussieck, and Mallik 
(2004) to include differential weighting of conflicts between pairs of commercials; while Garcia-Villoria and Salhi (2015) extend the framework of Bollapragada, Bussieck, and Mallik (2004) to include target rating point requirements. Alaei and Ghassemi-Tari (2011) consider the prime-time scheduling problem of a television network to maximize its revenue where the advertisers submit bids for time units, and propose a genetic algorithm based solutions. A generalization of this work has been considered by Ghassemi-Tari and Alaei (2013). Guerriero, Miglionico, and Olivito (2016) consider the scheduling problem of an Italian television network where the network decides whether to accept the scheduling requests of clients with both rating points and budget requirements, and propose several heuristics to solve the resulting problem.

A related stream of literature deals with scheduling movies in movie theaters to maximize revenues. Typical examples of such work are Swami, Eliashberg, and Weinberg (1999), Eliashberg et al. (2001), Somlo, Rajaram, and Ahmadi (2010), etc. We, however, note that scheduling is not the focus of our work. As a result we differ from this stream of literature in terms of both problem considered and the solution methodology employed.

The media revenue management literature on managing the inventory of rating points and/or slots for commercials is relatively sparse. Talluri and van Ryzin (2004) provide a brief account of the problem, while Phillips and Young (2012) provide a detailed practitioner-focused description of the problem summarizing the management challenges associated with the problem. Jones and Koehler (2002) are probably the first to formally study the upfront inventory allocation problem to maximize the revenue of a television network. They propose an auction mechanism where multiple buyers compete for the ad slots in the upfront market by submitting bids. They propose a heuristic solution to the resulting mixed integer program that provides a satisfactory solution within a short computation time. Both Bollapragada and Mallik (2008) and Araman and Popescu (2010) consider the inventory allocation problem between the upfront and the scatter markets. Bollapragada and Mallik (2008) consider the problem of a single, riskaverse, network that seeks to determine optimal amount of rating points to sell in the upfront to maximize its expected revenue subject to a chance constraint that revenue meets or exceeds a target value with a given probability. They describe sensitivity of the optimal solution with respect to the audience parameters and provide an explanation for the widely observed industry practice of selling $60-80 \%$ of the available inventory in upfront. Araman and Popescu (2010) consider a single network model as well where the network is risk neutral and maximizes the expected revenue by choosing the upfront rating points. They provide further operationalization of the problem by quantifying how the aggregate upfront rating points can be translated into customer level allocations under a multiplicative audience performance model.

While both Bollapragada and Mallik (2008) and Araman and Popescu (2010) study the single firm problem, our objective in this paper is to provide a competitive generalization of these two works by considering two television networks that compete in the upfront market. Our model, thus, is able to capture 
the strategic interaction between the two networks, and establish the unique Nash equilibrium. To the best of our knowledge, our paper is the first to consider competition in television revenue management problems.

\subsection{Supply Chain/Marketing Literature}

Our work has synergy with the research in supply chain and/or marketing where capacity and/or inventory is sold through two-stage processes (e.g., both futures and spot markets). Xie and Shugan (2001) are one of the firsts to comprehensively study the phenomenon of advance selling, where a seller allows buyers to purchase a good both before and during the consumption periods. The key assumption in their work is that the buyers are uncertain about the valuations of the product when they purchase it in advance, and that this valuation uncertainty is not resolved until the second period (consumption period). They show that this valuation uncertainty is the key driver of advance selling. They provide conditions under which a seller should advance sell and determine the optimal prices. Several papers have considered variations/extensions of Xie and Shugan (2001). For example, partially-refundable advance selling has been studied by Xie and Gerstner (2007), Guo (2009), and Gallego and Sahin (2010). The focus of these studies is how much refund, if any, should a firm offer to the customers. Advance selling under capacity constraint has been studied by Degraba (1995) and Yu et al. (2015). Advance selling under competition has been studied by Shugan and Xie (2005) and Cachon and Feldman (2017).

It is important to note that our work has fundamental differences with the cited works on advance selling. First, under advance selling, the buyers face an uncertain valuation of a product/service by purchasing it in advance. Such a scenario does not arise in our paper as the rating points sold during the upfront are guaranteed by the sellers. Second, under advance selling, the buys face uncertainty of valuations. In contrast, in our paper, the sellers face uncertainty about show performance, resulting in over- and underdelivery of rating points. Third, and unlike the literature on advance selling, the available capacity in our paper (i.e., the available rating points) is random. Finally, the focus of the papers on advance selling is whether and when advance selling is profitable/optimal for a seller. We, in contrast, take the current processes in the television industry as given, and seek to develop managerial insights about the process and the key decision variables. As a result our modeling approach and the resulting intuitions differ vastly from those in the advance selling literature.

Several researchers have studied advance booking discounts (ABD) in supply chains. The ABD contract between a retailer and a supplier typically has two wholesale prices, a discounted price for the inventory purchased before the selling season, and a regular price for replenishments during the selling season. Both Weng and Parlar (1999) and Tang et al. (2004) study the single-firm ABD problem while McCardle, Rajaram, and Tang (2004) provide a game theoretic model that provides a competitive generalization for the single-firm models. Chintapalli et al. (2017) explore the issue of coordinating supply chins through ABD contracts. Kleindorfer and Wu (2003) provide a survey of inventory management under 
such settings. $\mathrm{Wu}$ and Kleindorfer (2005) develop a two-stage model that integrates spot market transactions with supply chain contracting.

The cited works in two-stage inventory management typically consider demand uncertainty and seek to study supply contracts. The literature on ABD, similarly, considers demand uncertainty but ignores supply uncertainty. We differ from these works by considering both demand uncertainty (by letting the scatter market revenue be a function of random variables), supply uncertainty (by considering the performance uncertainty of the television shows). The audience uncertainty associated with our model is similar to yield uncertainty of the random yield literature. However, unlike the single-period random yield models, our work allows both over- and under-delivery on the rating points sold in upfront market. A reader is referred to Yano and Lee (1995) and Grosfeld-Nir and Gerchak (2004) for reviews of the random yield literature.

In summary, and to the best of our knowledge, this paper is the first to provide a game theoretic model of television revenue management. Our paper captures the strategic interactions between two television networks by explicitly modeling the uncertainties associated with show performances as well as with scatter market prices.

\section{THE MODEL}

Consider two competing television networks (henceforth referred simply as “networks”), $i$ and $j$, ( $i=1,2 ; j=3-i$ ), each interested in determining $X_{i}$, the aggregate amount of rating points inventory to sell in the upfront market to all customers. Table 1 at the end of Section 3 provides a summary of all notations used in the paper. The two networks (players) act non-cooperatively. The rating points of a particular show, and hence that of a commercial aired in a 30-second slot, on network $i, i=1,2$, is modeled ex ante as a positive random variable $\varepsilon_{i}$ defined over the interval $\left[A_{i}, B_{i}\right]$ with $B_{i} \geq A_{i} \geq 0$. Let $f_{i}\left(\right.$.) and $F_{i}($.) denote the probability density function and the cumulative distribution function of $\varepsilon_{i}$ respectively. We assume $F_{i}($. to be continuously differentiable. Let $\mu_{i}$ and $\sigma_{i}$ denote the mean and the standard deviation of $\varepsilon_{i}$ respectively. Inventory is assumed to be homogeneous, i.e., different ads of a network capture similar audiences. This assumption allows us to write the total rating points generated by network $i$ in $n_{i}$ slots simply as $n_{i} \varepsilon_{i}$. Araman and Popescu (2010) make an identical assumption to model the total audience and note that this multiplicative model corresponds to the stochastically proportional yield models used in random yield problems. Its main advantage is that it is simple to work with and is fairly general (see Yano and Lee, 1995). As explained in Section 1, in addition to choosing $X_{i}$, each network commits to airing commercials in $n_{i}$ slots out of the available $N_{i}$ slots during the broadcast year. Consistent with the practice that a television network does not change the number of commercial pods per hour of programming based on show performances, we assume $N_{i}, i=1,2$, to be known and exogenous. The upfront contract of each 
network guarantees the delivery of the promised rating points $X_{i}$ as well as the number of committed slots, $n_{i}$. The number of committed slots, $n_{i}$, is determined based on the agreed upon expected rating points of shows that a television network and its customers negotiate on. We let $v_{i}$ denote the agreed upon expected rating points of a single slot for network $i$. Therefore, $n_{i}=X_{i} / v_{i}$. Note that $v_{i}$ is not necessarily equal to $\mu_{i}$, the expected value of $\varepsilon_{i}$.

Any available rating point inventory not sold during the upfront is sold during the scatter market, which occurs throughout the broadcast season. The scatter price of network $i$, denoted by $p_{s i}$, is unknown ex ante. A network with hit shows, nevertheless, commands a higher scatter price compared to a network without hit shows. We model the scatter price of network $i$ as linearly increasing in the show performance $\varepsilon_{i}$, i.e., $p_{s i}=\theta_{i} \varepsilon_{i}$, where $\theta_{i}>0$ and is a given constant. Section 5.2 of our paper describes a generalization where we let the scatter price depend on the availability in the scatter market (thus, making $p_{s i}$ explicitly dependent on $X_{i}$ ). Recall from Section 1 that unlike the upfront market, no performance guarantee is offered by the networks during the scatter market and that the commercial time is sold on a per 30-second-slot basis. Thus, $p_{s i}$ in our model represents the price of a 30-second slot for network $i$. While a network can air ads that can be of either 15 -second or 30-second long, the 30-second is considered to be the standard unit for pricing, and that a 15-second slot is assumed to command half the price of a 30-second slot (Bollapragada and Mallik 2008). A network pays a penalty when the total realized rating points through the entire season, $N_{i} \varepsilon_{i}$, is not sufficient to meet the contracted rating points during the upfront market $X_{i}$. Let $g_{i} \geq 0$ be the penalty per rating point for not meeting the upfront commitments. To avoid a trivial solution, we will assume $g_{i} \geq \theta_{i}$, i.e., the penalty for each unfilled rating point is greater than the price coefficient of the rating points in the scatter market. The scatter market revenue of network $i, \pi_{s i}$, is given by:

$$
\pi_{s i}= \begin{cases}p_{s i}\left(N_{i}-X_{i} / v_{i}\right), & \varepsilon_{i} \geq v_{i} \\ p_{s i}\left(N_{i}-X_{i} / \varepsilon_{i}\right), & X_{i} / N_{i} \leq \varepsilon_{i} \leq v_{i} \\ -g_{i}\left(X_{i}-N_{i} \varepsilon_{i}\right), & \varepsilon_{i} \leq X_{i} / N_{i} .\end{cases}
$$

When $\varepsilon_{i} \geq v_{i}$, network $i$ is able to meet the upfront rating points commitment using fewer than $n_{i}=X_{i} / v_{i}$ slots. However, it must still offer the promised $n_{i}$ slots to customers, resulting in delivering more than promised. Thus, it will have only $\left(N_{i}-n_{i}\right)$ or $\left(N_{i}-X_{i} / v_{i}\right)$ slots to sell in the scatter market. When $X_{i} / N_{i} \leq \varepsilon_{i} \leq v_{i}$, network $i$ is unable to meet the upfront rating points commitment using the promised $n_{i}$ slots. Therefore, it must schedule additional slots (known as "make good" allocation in the industry) to meet the upfront commitment. It will then have $\left(N_{i}-X_{i} / \varepsilon_{i}\right)$ slots available for the scatter market. When 
$\varepsilon_{i}<X_{i} / N_{i}$, network $i$ must pay a penalty as the total rating points generated during the entire broadcast year is not enough to satisfy its upfront commitments.

We use Cournot competition to model the competition between the two television networks in the upfront market. We later show in Section 4.1 that choosing quantity is a dominant strategy for each network over choosing price. The two players choose their respective guaranteed rating points $X_{1}$ and $X_{2}$ simultaneously. Given $X_{1}$ and $X_{2}$, the price is set to clear the market. We use linear price functions of the following form:

$$
\begin{aligned}
& p_{1}=\alpha_{1}-\beta_{1} X_{1}-\gamma X_{2}, \\
& p_{2}=\alpha_{2}-\gamma X_{1}-\beta_{2} X_{2},
\end{aligned}
$$

where $\alpha_{i} \geq 0$ represents the maximum price clients are willing to pay network $i$ for one unit of rating point; $\beta_{i}, \geq 0$ represents the price reduction for one additional unit of network $i$ 's rating points supply; and $\gamma \geq 0$ captures the substitutability of rating points between the two networks. When $\alpha_{1}=\alpha_{2}$ and $\beta_{1}=\beta_{2}=\gamma$, the rating points of the two networks are perfect substitutes. We assume $\beta_{1} \beta_{2}-\gamma^{2}>0$ and $\alpha_{i} \beta_{j}-\alpha_{j} \gamma>$ 0 , for $i \neq j, i=1$, 2. By equations (2a) and (2b), the upfront price for network $i$ is inversely related to its as well as its competitor's rating points guarantee. Similar price functions are common in literature (see for example, Tsay and Agrawal 2000; Singh and Vives 1984). Our choice of Cournot competition to model the competition in the upfront market is consistent with literature. Both Bollapragada and Mallik (2008) and Araman and Popescu (2010) use quantity as the decision variable. In practice, television networks price upfront contracts based on the guaranteed deliverable rating points with a specified price per rating point (Bollapragada and Mallik 2008). Although both price and guaranteed rating points are specified in the contract, the guaranteed rating points are usually the starting point that networks need to commit and deliver-on first. Quantity competition, thus, is the appropriate choice to model competition in the upfront market. We, nevertheless, compare quantity strategy with price strategy in Section 4.2 and show that setting quantity indeed is a dominant strategy for each network.

Note that our formulation for upfront prices in equation (2a) and (2b) allow us to capture the relatively recent phenomena of time shifting and/or cord-cutting. Time shifting is said to occur when a viewer records a television show through a recording device (a DVR, for example) and watches at a later time; while cord-cutting refers to watching a television show using a streaming device over the internet. Whether to include the rating points generated through such activities towards counting the total rating points of a show is a hotly debated topic between a network and its customers (Steinberg 2016c). Steinberg (2017) further notes that television networks such as CBS in the United States has agreed to price 2017 upfront contracts using “total content ratings” specified by Nielsen Media Research, which includes livetelevision rating points, internet streaming, and time-shifted rating points for a period of 7 days after the airing of a show. The upfront price is still quoted in terms of price per thousand rating points; the only 
feature that changes is how the rating points are counted. Our modeling framework is general enough to accommodate these phenomena of time shifting and/or cord-cutting. Recall that the random variable $\varepsilon_{i}$ in our model denotes the GRP or the total rating points generated by a network during a broadcast year; while our decision variable $X_{i}$ represents the amount of rating points to sell in the upfront. In order to account for time-shifting and/or internet stream in our model, we will simply interpret $\varepsilon_{i}$ and $X_{i}$ to include time-shifted and internet-streamed rating points in our rating point counts.

The expected revenue of a network is the sum of upfront revenue and the expected scatter revenue. The networks choose respective upfront guaranteed rating points simultaneously and non-cooperatively. Thus, the expected revenue maximization problem of network $i, i=1$, 2, is given by:

$$
\begin{aligned}
& \operatorname{Max}_{X_{i}} \pi_{i}=p_{i} X_{i}+E\left[\pi_{s i}\right] \\
& =p_{i} X_{i}+ \\
& \int_{v_{i}}^{B_{i}} \theta_{i} \varepsilon_{i}\left(N_{i}-X_{i} / v_{i}\right) f_{i}\left(\varepsilon_{i}\right) d \varepsilon_{i}+\int_{X_{i} / N_{i}}^{v_{i}} \theta_{i} \varepsilon_{i}\left(N_{i}-X_{i} / \varepsilon_{i}\right) f_{i}\left(\varepsilon_{i}\right) d \varepsilon_{i}-g_{i} \int_{A_{i}}^{X_{i} / N_{i}}\left(X_{i}-N_{i} \varepsilon_{i}\right) f_{i}\left(\varepsilon_{i}\right) d \varepsilon_{i},
\end{aligned}
$$

where, $p_{1}$, and $p_{2}$ are given by equations (2a) and (2b) respectively. Our formulation in equation (3) can easily incorporate a discount factor for the scatter market profit by simply scaling the parameters $\theta_{i}$ and $g_{i}$ appropriately. Note that the inherent uncertainty of the show performances gives rise to the strategic behavior among the television networks (advertisers prefer to buy ad time on a network with hit shows). This uncertainty is highly significant in the upfront market but gets resolved rather quickly at the start of the scatter market. As a result, our formulation assumes competition in the upfront market but not in the scatter market. Using equation (3) and by setting $\partial \pi_{i} / \partial X_{i}=0, X_{i}$ is found to satisfy the following first order conditions for a given $X_{j}, j \neq i$ :

$$
\begin{aligned}
& \alpha_{i}-2 \beta_{i} X_{i}-\gamma X_{j}-\theta_{i}^{\prime}-\left(g_{i}-\theta_{i}\right) F_{i}\left(X_{i} / N_{i}\right)=0, \\
& \text { where, } \theta_{i}^{\prime}=\theta_{i} F_{i}\left(v_{i}\right)+\frac{\theta_{i}}{v_{i}} \int_{v_{i}}^{B_{i}} \varepsilon_{i} f_{i}\left(\varepsilon_{i}\right) d \varepsilon_{i}=\theta_{i}+\frac{\theta_{i}}{v_{i}} \int_{v_{i}}^{B_{i}}\left(1-F_{i}\left(\varepsilon_{i}\right)\right) d \varepsilon_{i} \geq \theta_{i} .
\end{aligned}
$$

The quantity $\theta_{i}^{\prime}$, in equations (4) and (5), is a constant that represents the expected loss of revenue in the scatter market from the unit increase of the upfront inventory $X_{i}$. When $X_{i}$ increases by one unit, the number of committed slots in the upfront increases by $\left(1 / v_{i}\right)$ units, which also tends to reduce the slots in the scatter market by $\left(1 / v_{i}\right)$ units and the scatter revenue by $\left(\theta_{i} / v_{i}\right)$ units. In addition, when rating is actually higher than $v_{i}$, the network cannot garner additional revenue from better performance, which is an additional opportunity cost to network $i$. Therefore, $\theta_{i}^{\prime}$ is greater than $\theta_{i}$. Replacing the terms $\alpha_{i}-2 \beta_{i} X_{i}-\gamma X_{j}$ in equation (4) by $p_{i}$ (per equations 2a, 2b), we note that $X_{i}$ satisfies $F_{i}\left(\frac{X_{i}}{N_{i}}\right)=\frac{p_{i}-\beta_{i} X_{i}-\theta_{i}^{\prime}}{g_{i}-\theta_{i}}$, which has a similar structure as that of the classic newsvendor solution. 
It is also worth noting that our model formulation in (1) and (3) assume that $X_{i} \leq N_{i} v_{i}$. Recall that $v_{i}$ is the agreed upon expected rating points for a slot between a network and its customers. Thus, $X_{i} \leq$ $N_{i} v_{i}$ simply implies that the amount of rating points sold by network $i$ in the upfront market does not exceed the total rating points it might generate during the entire broadcast season based on agreed upon rating points per slot. While it is mathematically possible to have $X_{i}>N_{i} v_{i}$, a rational network, in practice, will not choose to commit all available slots during the upfront. Bollapragada and Mallik (2008) report that it has been a common practice in the industry to sell about $60-80 \%$ of expected inventory during the upfront. Furthermore, we will later show that network $i$ has an incentive to specify a large $v_{i}$, which makes the relation $X_{i} \leq N_{i} v_{i}$ easier to hold mathematically. It could also be shown that $\alpha_{i}-2 \beta_{i} N_{i} v_{i}-\theta_{i}^{\prime}-\left(g_{i}-\right.$ $\left.\theta_{i}\right) F_{i}\left(v_{i}\right) \leq 0$ is a sufficient condition for $X_{i} \leq N_{i} v_{i}$ to hold. Therefore, for the remainder of the paper we will assume that the condition $X_{i} \leq N_{i} v_{i}$ holds.

Table 1: Summary of Notations

\begin{tabular}{|c|l|}
\hline Notation & Explanation \\
\hline$i, j$ & Index on the television networks, $i, j=1,2 ; j \neq i$ \\
\hline$\varepsilon_{i}$ & $\begin{array}{l}\text { Random variable denoting rating point of a single advertising slot of network } i, \\
\mu_{i}, \sigma_{i}: \text { mean and standard deviation of } \varepsilon_{i} \\
F_{i}: \text { CDF of } \varepsilon_{i}\end{array}$ \\
\hline$X_{i}$ & Upfront inventory choice of network $i$ (decision variable) \\
\hline$p_{i}, p_{s i}$ & Upfront and scatter prices, respectively, of network $i$ \\
\hline$\alpha_{i}, \beta_{i}, \gamma, \theta_{i}$ & Demand function parameters; $p_{i}=\alpha_{i}-\beta_{i} X_{i}-\gamma X_{j} ; p_{s i}=\theta_{i} \varepsilon_{i}$ \\
\hline$v_{i}$ & Agreed upon rating point of a slot for network $i$, \\
\hline$n_{i}$ & Number of slots committed by network $i$ during the upfront, $n_{i}=X_{i} / v_{i}$ \\
\hline$N_{i}$ & Total number of available slots for network $i$ \\
\hline$g_{i}$ & Penalty per rating point for not delivering on upfront commitments for network \\
\hline
\end{tabular}

\section{RESULTS AND INSIGHTS}

This section describes our results and insights. We first prove the existence of the unique Nash equilibrium and then proceed to develop insights about the equilibrium outcome. The following lemma describes our first result. 
Lemma 1: The best response function of network $i, X_{i}^{*}\left(X_{j}\right)$, decreases in $X_{j}$.

The proofs of all results are included in the Appendix. Lemma 1 indicates that the upfront inventories of the two networks, $X_{i}$ and $X_{j}$, are strategic substitutes. i.e., given one network commits more rating points during the upfront, the other network should commit less. This substitutability is captured by the parameter $\gamma$ in equations (2a) and (2b).

Proposition 1: There exists a unique Nash equilibrium to the upfront inventory game under Cournot competition.

Media revenue management is a highly complex problem and that the literature on media revenue management is relatively sparse. We are not aware of any work that studies competition in media/television revenue management. Planning for the overall upfront capacity ( $X_{i}$ in our model) is the most strategic decision a television network makes. Proposition 1 ensures the existence of unique equilibrium in this game. In addition to providing the theoretical basis for additional analyses, this result assures a rational network of the feasibility of scientific revenue management provided the other network is also rational.

\section{Proposition 2:}

(a) Network i's equilibrium upfront inventory $X_{i}^{*}$ increases in the parameters $\alpha_{i}, \beta_{j}, \theta_{j}, g_{j}, v_{i}$, and $N_{i}$, and decreases in the parameters $\alpha_{j}, \beta_{i}, \theta_{i}, g_{i}, v_{j}$, and $N_{j}$, for $i=1,2 ; j \neq i$.

(b) In particular, when the two networks are symmetric in parameters, the equilibrium upfront inventory $X_{i}^{*}$ increases in $\alpha, v$, and $N$, and decreases in the parameters $\beta, \theta, g$, and $\gamma$.

Proposition 2 describes the sensitivities of the equilibrium solution with respect to various problem parameters. The parameter $\alpha_{i}$ in our model (see equations 2a, 2b) represents customers' maximum willingness to pay. Per Proposition 2, the equilibrium upfront inventory of a network is increasing in its own parameter $\alpha_{i}$ and is decreasing in its competitor's. A customer's maximum willingness to pay in the upfront is often dictated by the potential viewership of a network's shows. Thus, a network with a strong lineup of shows (this might happen when a network has a stable lineup of hit shows for the upcoming broadcast season) should sell more in the upfront market while the competing network should sell less in the upfront. Per Bollapragada and Mallik (2008), NBC seems to be following this strategy. Our work, thus provides a theoretical validation for this strategy. The parameter $\beta_{i}$ represents the price sensitivity of the customers. Expectedly, the equilibrium upfront inventory of a network decreases in the price sensitivity parameter of its own customers and increases in the price sensitivity parameter of its competitor's customers. The scatter price in our model is dependent on show performance of a network. The parameter $\theta_{i}$ represents the scaling parameter for the scatter price. As $\theta_{i}$ increases, all else being equal, the scatter 
market becomes more attractive to network $i$. As a result, it reduces its equilibrium upfront inventory $X_{i}^{*}$. However, as elaborated in Lemma 1, the upfront inventories of the two networks are strategic substitutes. Therefore, as $X_{i}^{*}$ decreases, $X_{j}^{*}$ increases, implying $X_{j}^{*}$ increases with $\theta_{i}$. The scatter market price coefficient of one network, thus, influences the upfront inventory choice of both networks. Finally, it is intuitive that the equilibrium upfront inventory of a network is increasing in its total number of slots $N_{i}$ and the agreed upon expected rating points of a slot $v_{i}$.

Proposition 2(b) describes the sensitivities of the equilibrium outcome when the two television networks are symmetric in parameters. The sensitivity with respect to the maximum willingness to pay parameter $\alpha$ merits a discussion. A common practice in the television industry is to sell more in a "strong" upfront. Examples of such practices involving all major broadcast television networks have been reported by Chunovic (2003), while Steinberg (2005) reports a similar practice at ABC. Formally, upfront “A” is stronger than upfront " $\mathrm{B}$ " when $p_{i}^{A}\left(X_{i}, X_{j}\right) \geq p_{i}^{B}\left(X_{i}, X_{j}\right)$, for any given $X_{i}, X_{j}, i \neq j$. Under symmetric parameters, a strong upfront will be characterized by a higher value of the parameter $\alpha$. Per Proposition 2(b), selling more in a strong upfront is indeed an optimal practice. Our result, thus, is able to explain the observed practice. Interestingly, while studying a single firm problem, Bollapragada \& Mallik (2008) conclude that a network should sell less in a strong upfront for most realistic values of the problem parameters and that selling more in a strong upfront cannot be an optimal strategy. While our objective function is different from theirs, our competitive model is able to explain the observed practice under relatively mild assumption of symmetric parameters.

The effect of the parameter $\gamma$ on the equilibrium upfront inventory $X_{i}^{*}$ is ambiguous. However, Proposition 2(b) indicates that under the assumption of symmetric parameters, the equilibrium upfront inventory of a firm decreases in the parameter $\gamma$. A higher positive value of the parameter $\gamma$ represents higher substitution effect. Thus, as the inventory becomes more homogeneous, the equilibrium upfront price falls. A rational network responds to this by reducing the upfront inventory. Similarly, it could be shown that when that parameters of the two networks are symmetric, the equilibrium upfront inventory $X_{i}^{*}$ is increasing in total number of available slots $N$.

Proposition 3: Network i's equilibrium expected profit is increasing in $v_{i}$, and is decreasing in $v_{j}$, i.e., $\pi_{i}\left(X_{i}^{*}, X_{j}^{*}\right) / \partial v_{i} \geq 0$, and $\partial \pi_{i}\left(X_{i}^{*}, X_{j}^{*}\right) / \partial v_{j} \leq 0$, for $i=1,2 ; j \neq i$.

Recall that $v_{i}$ is the agreed upon expected rating points for a slot, which is not necessarily the same as $\mu_{i}$, the mean of the rating points distribution. Proposition 3 indicates that a network has incentive to exaggerate rating points of its shows, as the equilibrium expected profit is increasing in $v_{i}$. Why does this happen? As elaborated in Section 1, the upfront contract, while priced exclusively based on deliverable rating points, 
promises a certain number of slots as well. When the shows of a network perform better than expected, the promised number of rating points will likely be met by fewer than the promised number of slots. The network, however, is contractually obligated to offer its customers the promised number of slots. This results in a loss of revenue as the network over-delivers on the rating points without any additional revenue. Exaggerating show performances results in fewer number of promised slots. This reduces the probability of over-delivery and that any shortfall in rating points can be made up using “make good” allocations.

Shows performing better than expected are not uncommon in the television business. During the 2014-2015 broadcast year, both "Gotham” on FOX and "Scorpion” on CBS have reportedly performed much better than expected (Collins, 2014). Similarly, “Apprentice” on NBC or "Desperate Housewives” on $\mathrm{ABC}$ performed much better than expected during their respective opening seasons, resulting in a loss of revenue. It is indeed a well-established practice to inflate the show performances in the television industry. Proposition 3, thus, provides an explanation for the observed practice. Note, however, that per Proposition 2, as $v_{i}$ increases, so does the equilibrium rating points guarantee $X_{i}^{*}$. As a result, exaggeration of show performances has only limited potential for reducing the probability of over-delivery and/or improving equilibrium profit.

We study the effect of variance of show performance on the equilibrium upfront inventory next. To accomplish this, we define a new random variable $\varepsilon_{\tau i}=\left(1-\tau_{i}\right) \mu_{i}+\tau_{i} \varepsilon_{i}$, where $0 \leq \tau_{i} \leq 1$ is a constant, to replace the random variable $\varepsilon_{i}$ representing the show performances. Note that by definition, $\varepsilon_{\tau i}=\mu_{i}$ when $\tau_{i}=0$, and $\varepsilon_{\tau i}=\varepsilon_{i}$ when $\tau_{i}=1$; and that $E\left(\varepsilon_{\tau i}\right)=\mu_{i}, \operatorname{Var}\left(\varepsilon_{\tau i}\right)=\tau^{2} \operatorname{Var}\left(\varepsilon_{i}\right)$. Thus, $\varepsilon_{i}$ is a special case of the new random variable $\varepsilon_{\tau i}$, and that the variance of $\varepsilon_{\tau i}$ increases as $\tau_{i}$ increases. Therefore, the parameter $\tau_{i}$ is an indicator of variability of show performances. The purpose of defining $\varepsilon_{\tau i}$ is to parameterize the uncertainty effect of show performances while preserving the mean effect of that uncertainty. As Gerchak and Mossman (1992) point out, the benefit of adopting this mean-preserving parameterization is that it provides a mechanism for exploring changes resulting from exogenous changes in uncertainty. A similar parameterization can also be found in Li and Atkins (2002) and in Song et al. (2008). The following proposition describes our result.

Proposition 4: If $v_{i} \geq \mu_{i}$ and $\alpha_{i}-2 \beta_{i} \mu_{i} N_{i}-\tau_{i} \theta_{i}^{\prime}-\left(g_{i}-\theta_{i}\right) F_{i}\left(\mu_{i}\right) \leq 0$, then $X_{i}^{*}$ decreases in $\tau_{i}$ and increases in $\tau_{j,} i, j=1,2$.

Under a set of sufficient conditions, the equilibrium upfront inventory of a network is decreasing in its variance of shows, and is increasing in its competitor's show variance. The sufficient conditions in the proposition ensures that $X_{i}^{*} \leq N_{i} \mu_{i}$, or the equilibrium upfront inventory choice of a network cannot exceed the total expected rating points for the entire season. In our numerical experimentation we find that a wide 
range of parameters that satisfy the condition $X_{i}^{*} \leq N_{i} \mu_{i}$. The uncertainty of the show performance is likely to be high when a network starts a new broadcast year with many new shows. In practice, many television shows run across multiple seasons. In fact there are examples in recent years when one network has entered a broadcast year with few new shows while other networks have had new shows. Under such a scenario, per Proposition 4, the network with new shows should sell less in the upfront, while a network with a relatively stable lineup should sell more in the upfront as its inventory is a strategic substitute of the inventory of the other network. The result is intuitive. When show uncertainties are high, saving inventory for the scatter allows a network to reduce the chances of over-delivery or paying a penalty on the promised upfront rating points.

We next turn to a numerical study to derive insights about how other parameters of our model affect the equilibrium upfront inventory as well as the equilibrium expected profit.

\section{Numerical Study 1}

We conduct numerical experiments involving a symmetric game with two competing television networks. The baseline parameters for the numerical study, for $i=1,2$, are as follows: $N_{i}=18,000 ; \alpha_{i}=$ $\$ 100$ per one thousand rating points; $\beta_{i}=\$ 10^{-12}$ per rating point; $\gamma=\$ 0.60 \times 10^{-12}$ per rating point; $\theta_{i}=\$ 0.03$ per one thousand rating points; $g_{i}=\$ 80$ per one thousand rating points. The random variable $\varepsilon_{i}$, denoting the show performance, is assumed to follow normal distribution with baseline parameters $\mu_{i}=$ 1500,000 and $\sigma_{i}=450,000$ rating points. Phillips and Young (2012), in Tables 11.1 and 11.2 of their work, provide estimates of upfront prices for thousand rating points for several television networks, including ABC, CBS, FOX, and NBC. Our baseline parameter values are chosen to be consistent with those provided by Phillips and Young (2012).

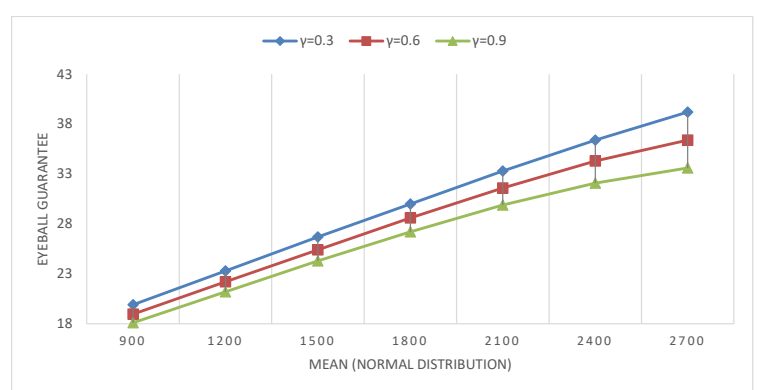

Figure 1(a): Plot of equilibrium upfront guarantee $\left(X^{*}\right)$ vs. the mean of $\varepsilon_{i}$ (normal distribution)

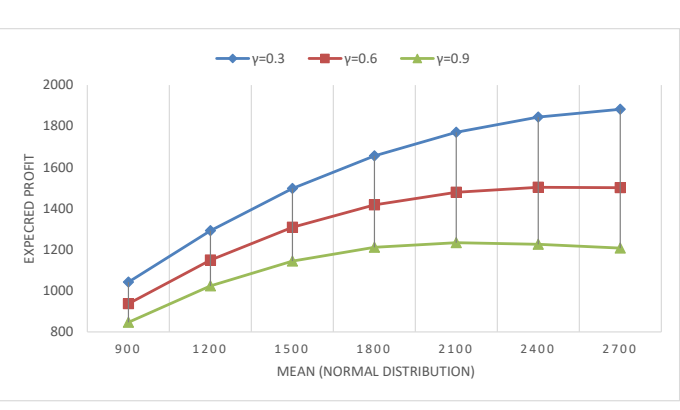

Figure 1(b): Plot of equilibrium expected profit vs. the mean of $\varepsilon_{i}$ (normal distribution) 


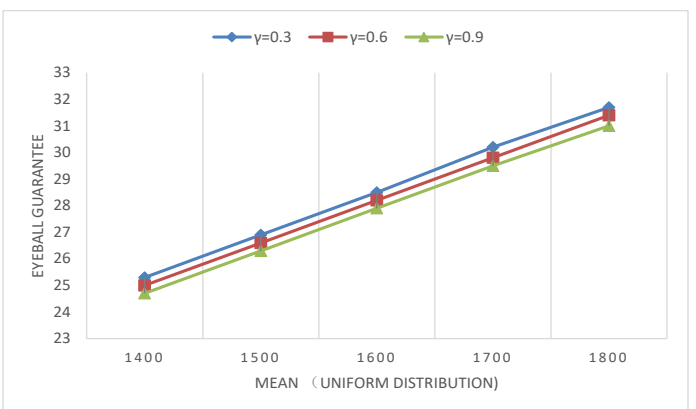

Figure 2(a): Plot of equilibrium upfront guarantee $\left(X^{*}\right)$ vs. the mean of $\varepsilon_{i}$ (uniform distribution) Figure 1(a) above shows how the equilibrium upfront inventory (or the guaranteed rating points) changes with respect to the mean of the show performances $\mu_{i}$ for different values of the substitutability parameter $\gamma$, while Figure 1(b) shows how the expected profit of a network changes with respect to $\mu_{i}$. The random variable for show performance is assumed to be normal for these plots with parameter values set at their respective baseline values. Per Figure 1(a), the equilibrium upfront inventory increases with the mean, for a given standard deviation. The result is intuitive: a higher mean of show performance allows a network to offer more inventory in the upfront. Figure 1(a) also shows that for a given mean of show performance, the upfront inventory is decreasing in $\gamma$. This is consistent with Proposition 2(b) as the upfront inventories of the two networks are strategic substitutes. Per Figure 1(b), for relatively low value of $\gamma$, the equilibrium expected profit of a network is non-decreasing in the mean of show performances. However, a similar claim cannot be made when $\gamma$ is relatively high (for $\gamma=0.6$ or 0.9 ). As the mean of show performance increases, so does the equilibrium upfront inventory, and the upfront price falls consequently. The price reductions become significant at relatively higher values of $\gamma$, resulting in a lower expected profit.

To study the sensitivity of the results in Figures 1(a) and 1(b) with respect to the normal distribution assumption, we have repeated our numerical study under the uniform distribution assumption of show performances. Figures 2(a) and 2(b) describe our results. To vary the mean of the uniform distribution while keeping the variance constant, we have varied the range of the uniform distributions. For example, to obtain a mean of 1500000, we have considered the uniform distribution over the range [1400000, 1600000]; while to obtain a mean of 1600000 , we have considered the uniform distribution over the range [1500000, 1700000]; etc. Figures 2(a) and 2(b) are qualitatively similar to Figures 1(a) and 1(b) respectively. Finally, it is worthwhile to mention that qualitative nature of the plots in the Figures 1(a) and 1(b) remain unchanged when plotted for different values of the parameter $v_{i}$, the agreed upon expected rating of a show.

Under a set of sufficient conditions, Proposition 4 describes the sensitivity of the equilibrium upfront inventory with respect to $\tau_{i}$, which is a measure of the variability of show performances. We conclude Numerical Study 1 with a discussion about how $\sigma$, the standard deviation of show performance, might affect the equilibrium upfront inventory and the expected profit of a network. Figures 3(a) and 4(a) 
below describe the sensitivity of the equilibrium upfront inventory with respect to the standard deviation of the show performance for normal and uniform distributions respectively. The results are intuitive and are consistent with Proposition 4: a higher variably of show performance induces a network to reduce the upfront inventory. Figures 3(b) and 4(b) below describe the sensitivity of the equilibrium expected profit with respect to the standard deviation of the show performance for normal and uniform distributions respectively. The plots show that an increase in the variability of the show performance reduces the equilibrium expected profit; and that for a given standard deviation, the expected profits are lower when the shows of the two networks are close substitutes (higher values of $\gamma$ ).

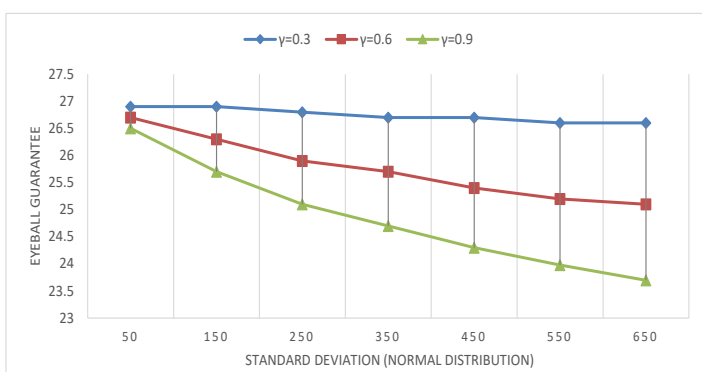

Figure 3(a): Plot of equilibrium upfront guarantee $\left(X^{*}\right)$ vs. the standard deviation of $\varepsilon_{i}$ (normal distribution)

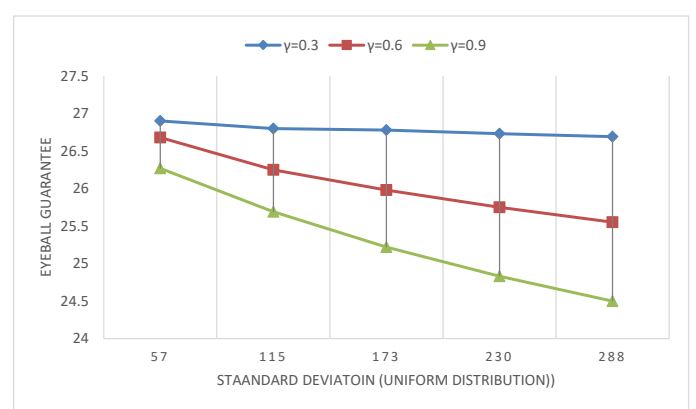

Figure 4(a): Plot of equilibrium upfront guarantee $\left(X^{*}\right)$ vs. the standard deviation of $\varepsilon_{i}$ (uniform distribution)

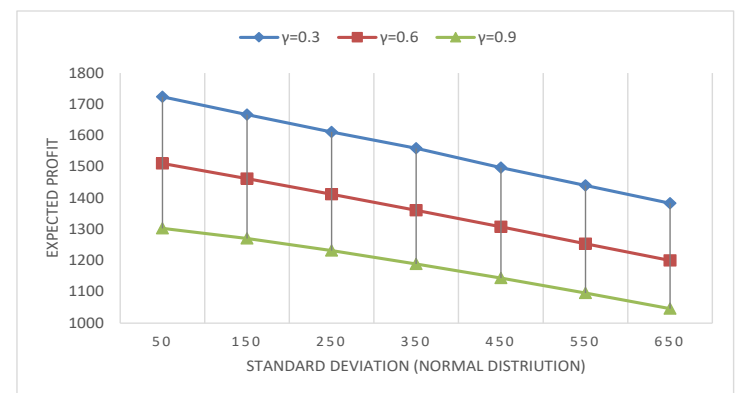

Figure 3(b): Plot of equilibrium expected profit vs. the standard deviation of $\varepsilon_{i}$ (normal distribution)

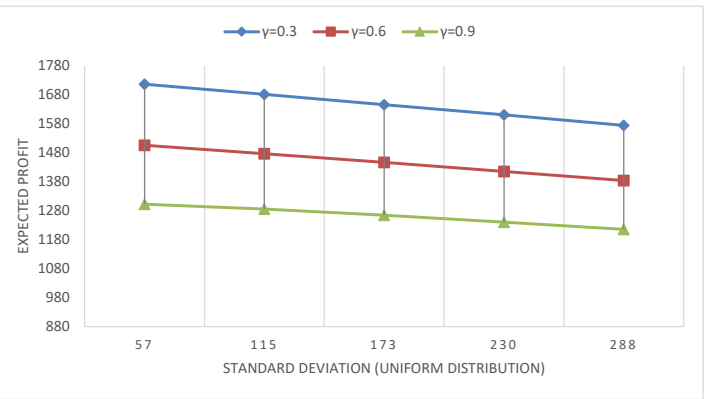

Figure 4(b): Plot of equilibrium expected profit vs. the standard deviation of $\varepsilon_{i}$ (uniform distribution)

\subsection{Lack of Transparency, Quantity Competition, and Price Competition}

Consistent with the literature, our analysis so far assumes a Cournot competition, where each network sets a quantity (i.e., the upfront rating points inventory) and the price is determined endogenously to clear the market. In reality, the upfront inventory allocation is a highly complex process that often lacks transparency (Vranica 2006). Vranica (2006) further states, “Networks keep advertisers in the dark about demand, leaving media buyers to pick up scraps of information as they bargain. Prices are often based on what an advertiser has paid in the past, rather than purely on demand.” Numerous articles in the popular 
business press confirm this reality for the 2016 upfront as well (see, for example, Shields, 2016). Under such an environment a pure quantity competition model may not be a completely realistic representation of the upfront market. Our objective in this paper is to develop as much insight as possible about the competitive upfront inventory allocation problem. As a result, we consider following four possible models in this subsection:

- Model Q, where network 1 chooses quantity (rating points), while network 2 chooses price;

- Model P, where network 1 chooses price, while network 2 chooses quantity (rating points);

- Model B, where both networks choose price (the Bertrand model); and

- Model C, where both networks choose quantity (the Cournot model).

Our discussions so far have focused on Model $\mathbf{C}$ above, or on the Cournot model. If the networks are involved in a Bertrand competition (Model B), then each firm will maximize their expected profit by choosing the upfront prices $p_{1}$ and $p_{2}$. Using equations (2a) and (2b), the resulting upfront inventories (or the guaranteed rating points) are as follows:

$$
\begin{aligned}
& X_{1}=a_{1}-b_{1} p_{1}+c p_{2}, \\
& X_{2}=a_{2}-b_{2} p_{2}+c p_{1},
\end{aligned}
$$

where, $a_{i}=\left(\alpha_{i} \beta_{j}-\alpha_{j} \gamma\right) /\left(\beta_{i} \beta_{j}-\gamma^{2}\right), b_{i}=\beta_{i} /\left(\beta_{i} \beta_{j}-\gamma^{2}\right)$, and $c=\gamma /\left(\beta_{i} \beta_{j}-\gamma^{2}\right)$.

The formulations for other two models, $\mathbf{P}$ and $\mathbf{Q}$, are similar. For example, in model $\mathbf{P}$, network 1 maximizes its expected profit by determining price $p_{1}$ given networks 2's decision of $X_{2}$. By rewriting equation (2a), Network 1's inventory, consequently, is given by

$$
X_{1}=\left(\alpha_{1}-\gamma X_{2}-p_{1}\right) / \beta_{1} .
$$

Network 2 maximizes its expected profit by determining $X_{2}$ given network 1 's decision of $p_{1}$. By rewriting equation (7), Network 2's price $p_{2}$ is given by

$$
p_{2}=\left(a_{2}+c p_{1}-X_{2}\right) / b_{2} \text {. }
$$

Following an approach similar to that of the proof of Proposition 1, it could be shown that unique Nash equilibrium exists for each of three games described by models $\mathbf{Q}, \mathbf{P}$, and $\mathbf{B}$. The proofs of these existence and uniqueness results are available from the authors upon request. The existence of unique equilibrium for all four models allows us to prove the following result.

\section{Proposition 5:}

(a) Choosing quantity in the upfront market is the dominant strategy of a network irrespective of its competitor's actions, i.e., the Cournot game is equilibrium outcome when the networks are free to choose either price or quantity.

(b) When the two networks are symmetric in parameters, the Cournot game results in less upfront inventory, higher upfront prices, and higher expected profit than the Bertrand game. 
Per Proposition 5, setting quantity is the dominant strategy for each network. As a result, the Cournot game is the dominant equilibrium outcome. A television network, thus, is better off choosing quantity (or the rating points inventory) independent of the choice of its competitor. Interestingly, this is always not the case in practice. There are reported instances where a television network has had negotiated prices with its clients. A recent article in the Variety magazine (Steinberg, 2016b) about the 2016 upfront notes the following.

Indeed, the networks and ad buyers have been at loggerheads for several weeks over the rate of increase that ought to be paid. Initial bids from the networks called for double-digit CPM increases - some as much as 15\% or higher, according to ad buyers. Advertisers, meanwhile, have expressed a desire to pay CPM increases of just 4\% to 5\%. Many buying executives have made a hard point of not wanting to agree to increases of more than 9\%, though one of the people familiar with discussions said that CBS pressed for some agreements that call for hikes in the low double-digit percentage range.

The above statements seem to suggest that the networks are engaging in a price competition, rather than a quantity competition. Per Proposition 5, this cannot be an optimal strategy for either network. A network will be better off choosing quantity irrespective of its competitor's choice. In fact, as Proposition 5(b) states, under the assumption of symmetric parameters, both networks will command higher prices and higher expected profits in quantity competition than in price competition. Our work, thus, can serve as a useful prescriptive guideline to practitioners planning the upfront strategy of a network.

Another practice that seems to be prevalent among the networks is to engage in intense price negotiation during the upfront that follows a strong show performance of a network during the previous year. The CW network, following a strong performance of its sci-fi and superhero shows during the 20142015 broadcast year, and stable lineup for the shows in the following year, reportedly was successful in negotiating a higher price hike than any other broadcast network during the 2015 upfront (Steinberg 2016a). CBS reportedly followed a similar course following strong performance of its "CSI” franchise during 2004 (Lafayette and Friedman, 2004). Our work, admittedly, does not consider multiple time periods. However, strong performance and a stable line-up of shows can be mapped into our modeling framework by considering high mean and low variance of the random variable $\varepsilon_{i}$, representing the show performance. Note, that the result in Proposition 5 is independent of the parameters representing show performance. The advantage of setting quantity over price is inherent in its structure, rather than the choice of the problem parameters. In fact, Singh and Vives (1984) proves a similar result in a stylized economic model involving deterministic demand. Our work, thus, provides the following guideline to a practitioner: independent of the expectations of show performances, a network is better off choosing quantity over price. Proposition 5 
justifies our choice of Cournot competition as the framework for this paper. We will, for the remainder of this paper, consider only Cournot competition.

\subsection{Comparison with a Centralized/Single Firm Case}

As elaborated earlier, the key contribution of our work is to consider competition in television revenue management. Our work thus far considers a non-cooperative game where each network maximizes its own expected revenue. We now compare our results with a centralized/single firm scenario. To make our results directly comparable with a single-firm scenario, we will assume that an imaginary central planner maximizes the total expected profit of the two networks, $i$ and $j$, by jointly determining $X_{i}$ and $X_{j}$, i.e., $\underset{X_{i}, X_{j}}{\operatorname{Max}} \pi=p_{i} X_{i}+E\left[\pi_{s i}\right]+p_{j} X_{j}+E\left[\pi_{s j}\right]$. All parameters, notations, and assumptions developed thus far will continue to remain valid. Additionally, we will let $X_{i}^{0}, X_{j}^{0}$, and $\pi^{o}$ denote the optimal upfront inventories and the optimal profit, respectively, in the single firm scenario. The following proposition describes our result.

Proposition 6: When the parameters of the two network are symmetric,

(a) the single firm scenario delivers fewer rating points in the upfront market than the Cournot game, i.e., $X_{i}^{0}<X_{i}^{*}, i=1,2$.

(b) when the distribution of show performances $\varepsilon_{i}$ is uniformly distributed or is normally distributed with $\gamma>\frac{g-\theta}{\sqrt{2 \pi} N \sigma}$, the rating points difference $\left(X_{i}^{*}-X_{i}^{0}\right)$ increases in the parameters $v, \alpha$, $\gamma$ and decreases in the parameters $\beta$ and $g$.

Note that our construct of centralized/single firm case can also be viewed as a cooperative game between the two networks. Proposition 6 indicates that competition, expectedly, results in delivery of a higher upfront inventory than the centralized case. With mild restrictions on some common distributions, we further find that the difference between the upfront inventories of the competitive and centralized cases increases in the parameters $v, \alpha, \gamma$, and decreases in the parameters $\beta$ and $g$. As $v$ increases, the equilibrium upfront inventory $X_{i}^{*}$, per Proposition 2(b), also increases. It could be shown that the optimal upfront inventory $X_{i}^{0}$ under the centralized case also increases in the parameter $v$. However, the rate of increase of the former is higher than that of the latter, resulting in a higher $\left(X_{i}^{*}-X_{i}^{0}\right)$. A similar intuition holds for the sensitivity result with respect to the parameter $\alpha$. As the unit penalty $g$ increases, the upfront market becomes potentially less attractive and that the equilibrium upfront inventory decreases under both centralized and competitive settings. However, the centralized system, per Proposition 6(a), offers less upfront inventory to start with. Therefore, the inventory reduction in the centralized system is lower 
compared to the competitive setting for an identical increase in the unit penalty cost. The net effect of this is a reduction in $\left(X_{i}^{*}-X_{i}^{0}\right)$. The price sensitivity parameter $\beta$ has a similar effect.

To develop additional insights about the centralized and the competitive models we conduct a numerical study next.

\section{Numerical Study 2}

Our objective in this numerical study is to develop insights about sensitivities of $\left(X_{i}^{*}-X_{i}^{0}\right)$, the difference in equilibrium upfront inventory, and $\pi^{0}-\left(\pi_{i}^{*}+\pi_{j}^{*}\right)$, the difference in equilibrium profit, with respect to the mean and the standard deviation of the show performance. The numerical values of the problem parameters used in this study are identical to those of Numerical study 1. Figures 5(a) and 5(b) plot, respectively, $\left(X_{i}^{*}-X_{i}^{0}\right)$ and $\pi^{0}-\left(\pi_{i}^{*}+\pi_{j}^{*}\right)$ against the mean of show performance under normal distribution assumption, while Figures 6(a) and 6(b) plot the same quantities under the assumption of uniform distribution. The sensitivities of $\left(X_{i}^{*}-X_{i}^{0}\right)$ and $\pi^{0}-\left(\pi_{i}^{*}+\pi_{j}^{*}\right)$ with respect to the standard deviation of show performance, under normal and uniform distribution assumptions, are described in Figures 7(a), 7(b), 8(a), and 8(b) respectively. We note that the qualitative nature of the plots remain unchanged between the normal and the uniform distribution, indicating that our findings are quite robust. As the mean or the standard deviation of the show increases, both $\left(X_{i}^{*}-X_{i}^{0}\right)$ and $\pi^{0}-\left(\pi_{i}^{*}+\pi_{j}^{*}\right)$ increase. However, for a given mean of show performance, the inventory and profit differences between the two models are higher for higher values of the substitutability parameter $\gamma$. This indicates that the benefit of coordination is higher when the shows of the two networks are closer substitutes.

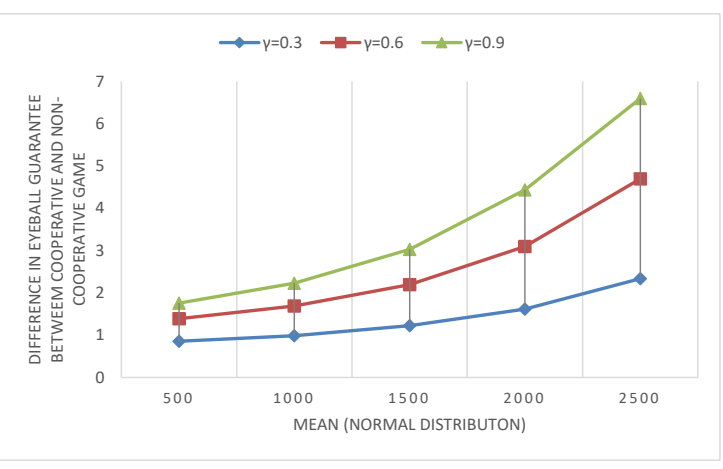

Figure 5(a): Plot of $\left(X^{*}-X^{0}\right)$ vs. the mean of $\varepsilon_{i}$ (normal distribution)

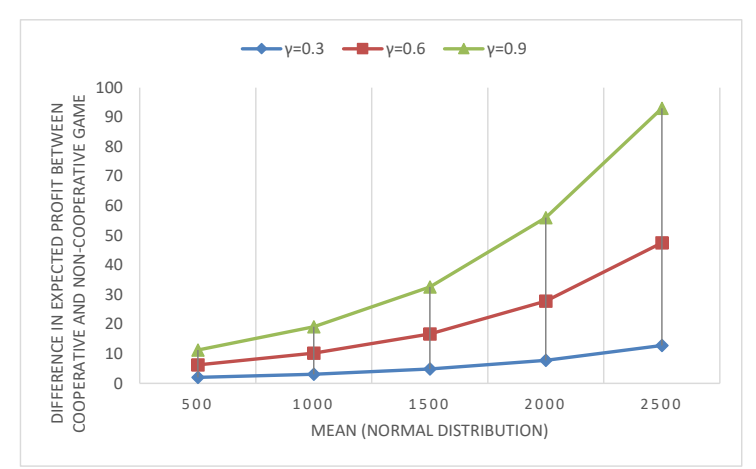

Figure 5(b): Plot of $\pi^{0}-\left(\pi_{i}^{*}+\pi_{j}^{*}\right)$ vs. the mean of $\varepsilon_{i}$ (normal distribution) 


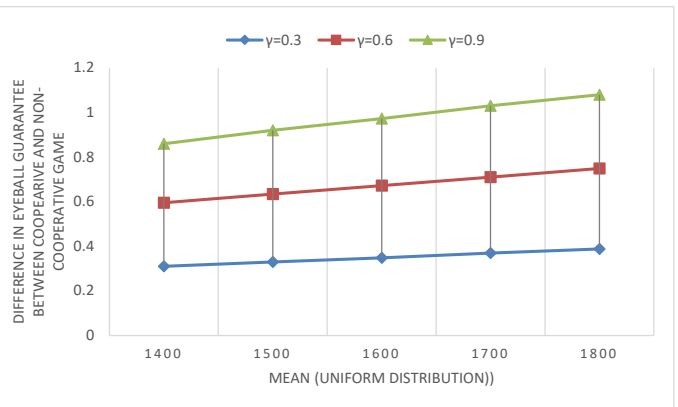

Figure 6(a): Plot of $\left(X^{*}-X^{0}\right)$ vs. the mean of $\varepsilon_{i}$ (uniform distribution)

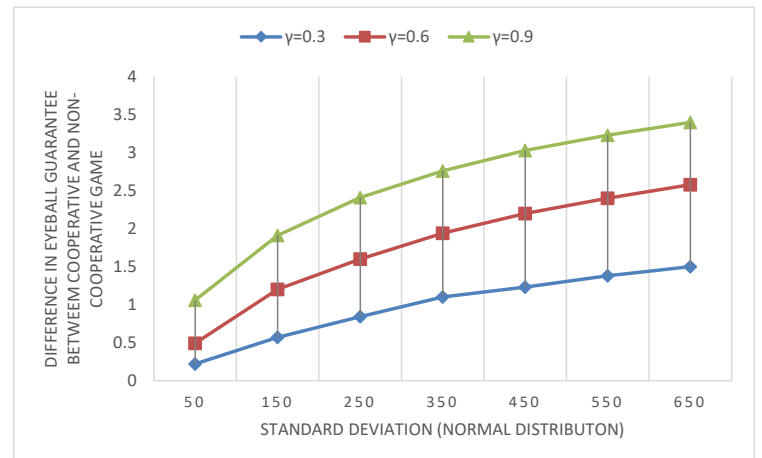

Figure 7(a): Plot of $\left(X^{*}-X^{0}\right)$ vs. the standard deviation of $\varepsilon_{i}$ the (normal distribution)

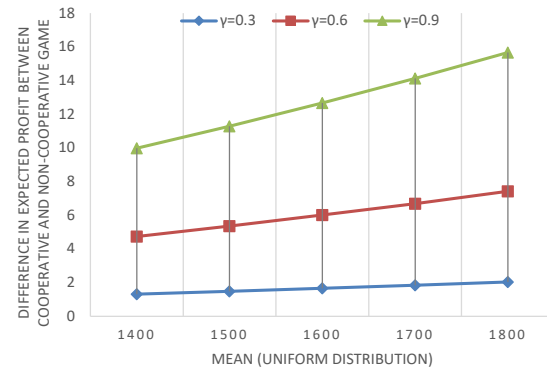

Figure 6(b): Plot of $\pi^{0}-\left(\pi_{i}^{*}+\pi_{j}^{*}\right)$ vs. the mean of $\varepsilon_{i}$ (uniform distribution)

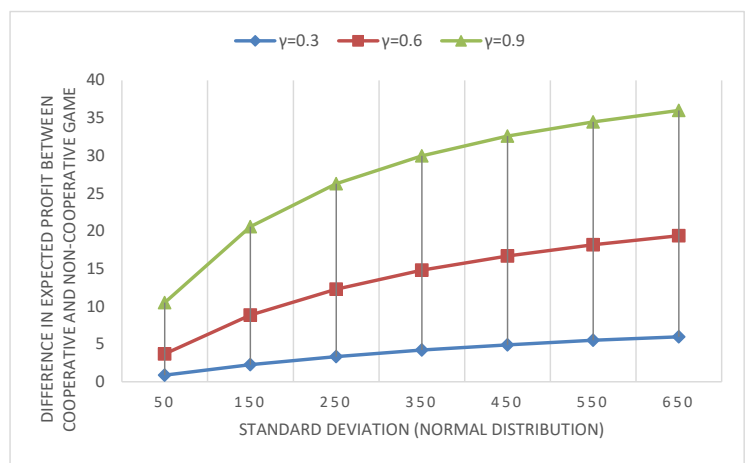

Figure 7(b): Plot of $\pi^{0}-\left(\pi_{i}^{*}+\pi_{j}^{*}\right)$ vs. standard deviation of $\varepsilon_{i}$ (normal distribution)

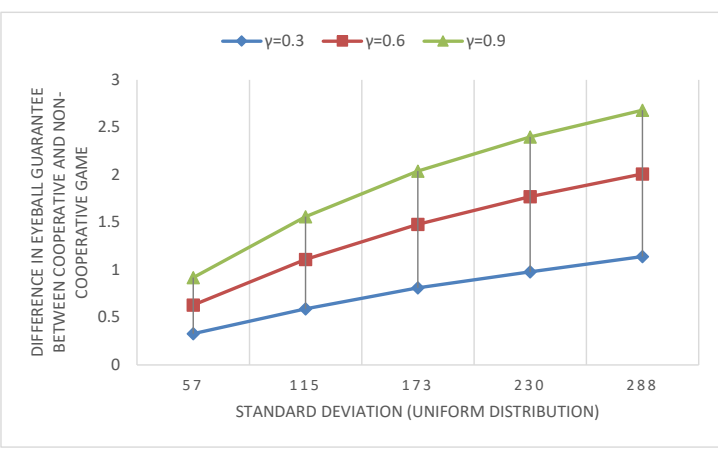

Figure 8(a): Plot of $\left(X^{*}-X^{0}\right)$ vs. the vs. standard deviation of $\varepsilon_{i}$ (uniform distribution)

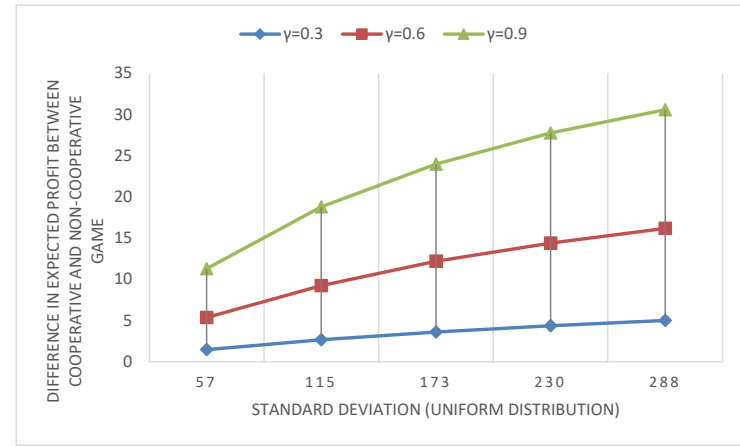

Figure 8(b): Plot of $\pi^{0}-\left(\pi_{i}^{*}+\pi_{j}^{*}\right)$ vs. standard deviation of $\varepsilon_{i}$ (uniform distribution)

\section{EXTENSIONS}


Our analyses so far describes the high level decision facing a television network: how much inventory to sell during the upfront on an aggregate basis to all its clients (or customers). We describe three extensions of our basic model in this section. The first extends our analyses to individual client level, the second models availability-dependent scatter market price, while the final extension models information asymmetry between the two television networks. These three extensions are described in Sections 5.1, 5.2, and 5.3 respectively. We note that the three extensions are independent of each other, i.e., our analyses of multiple clients do not assume availability-dependent scatter price or information asymmetry, etc.

\subsection{Multiple Clients Analysis}

Our model so far considered the most strategic decision in media revenue management: planning for the aggregate capacity in the upfront market. This section extends the analysis to multi-client setting. During the upfront market, multiple clients approach each network around the same time (usually over a short time period of 2-3 weeks), and contracts are negotiated in parallel. Suppose there are $K$ clients, and let $X_{k i}(k=1,2, \ldots, K ; i=1,2)$ be the network $i$ 's rating points guarantee for the $k^{\text {th }}$ client. Assume that the upfront price per rating point charged by network $i$ is given by: $p_{k i}=\alpha_{k i}-\beta_{k i} X_{k i}-\gamma_{k} X_{k j}$. Our price function assumes that the maximum willingness to pay $\alpha_{k i}$ and the sensitivity parameter $\beta_{k i}$ varies across the clients of a network, while the substitutability parameter $\gamma_{k}$ does not. To ensure that the demand function of the multiple clients case matches that of the aggregate case discussed thus far, we assume $\frac{\sum_{k=1}^{K} \alpha_{k i}}{K}=\alpha_{i}, \beta_{k i}=K \beta_{i}$, and $\gamma_{k}=K \gamma$. The assumption $\frac{\sum_{k=1}^{K} \alpha_{k i}}{K}=\alpha_{i}$ implies that the clients' maximum willingness to pay in the aggregate planning problem is the average of each individual client's maximum willing to pay. Consistent with the practice, our formulation allows a network to charge different prices to different clients. The assumptions $\beta_{k i}=K \beta_{i}$ and $\gamma_{k}=K \gamma$ indicate that the impact of an additional unit of supply for one client on price in the multi-client case is equivalent to that of $K$ additional units of supply in the aggregate planning problem. For the ease of exposition and to facilitate the comparison with the aggregate planning results, we assume other parameters $g_{i}, v_{i}, \theta_{i}$, are the same across the clients of a network, and are identical to those of the aggregate planning problem described in Section 3 of the paper. The expected revenue maximization problem of network $i, i=1,2$, is as follows:

$$
\begin{aligned}
\max _{X_{1 i}, x_{2 i}, \ldots, x_{k i}} \pi_{i}= & \operatorname{Max} \sum_{k=1}^{K} \pi_{k i} \\
= & \sum_{k=1}^{K} p_{k i} X_{k i}+\int_{v_{i}}^{B_{i}} \theta_{i} \varepsilon_{i}\left(N_{i}-\frac{\sum_{k=1}^{K} X_{k i}}{v_{i}}\right) f_{i}\left(\varepsilon_{i}\right) d \varepsilon_{i}+\int_{\sum \frac{X_{k i}}{N_{i}}}^{v_{i}} \theta_{i} \varepsilon_{i}\left(N_{i}-\frac{\sum_{k=1}^{K} X_{k i}}{\varepsilon_{i}}\right) f_{i}\left(\varepsilon_{i}\right) d \varepsilon_{i} \\
& -g_{i} \int_{A_{i}}^{\sum_{N_{i}}^{\frac{X_{k i}}{N_{i}}}}\left(\sum_{k=1}^{K} X_{k i}-N_{i} \varepsilon_{i}\right) f_{i}\left(\varepsilon_{i}\right) d \varepsilon_{i}, \text { for } i=1,2 .
\end{aligned}
$$


Following a procedure similar to those described in the proofs of Lemma 1 and Proposition 1, it could be shown that a unique Nash equilibrium exists in the multi-client revenue management game.

Proposition 7: The total equilibrium upfront inventory under multiple clients scenario equals the equilibrium upfront inventory under aggregate planning, i.e., $X_{i}^{*}=\sum_{k=1}^{K} X_{k i}^{*}$

In practice, a television network often quotes a different upfront price to each client. There could be several reasons behind this, including, the lack of transparency, the size of client's ad budget, etc. Per Proposition 7, even when each client is charged a different price, a television network can simply add the optimal clientlevel upfront inventory allocations to obtain the aggregate upfront inventory and still be assured of the highest expected profit. Alternatively, a television network can, ex ante, solve aggregate planning problem by simply assuming that one large client will buy the entire upfront capacity paying a price that equals the average per rating point paid by its clients. Proposition 7, thus, allows a network to simplify its upfront planning problem considerably. What drives this result? Under multiple client upfront problem, a television network bears the risk of over-delivery or penalty for each client separately. This eliminates the possibility of risk pooling across clients, i.e., the scenario where the excess from one client could have been used to compensate the penalty for another client. It is worthwhile to mention that Araman and Popescu (2010) concludes that (see their Proposition 4) a single television network should divide its aggregate upfront capacity equally across all its clients to achieve optimal allocation. They ascribe this result to their assumption of uniform penalty cost across clients. While our work is not directly comparable to theirs because of differing model setup and assumptions, Proposition 7 can still be viewed as a generalization of the corresponding result of Araman and Popescu (2010). Unlike theirs, we allow a television network to charge different prices to different clients, and to allocate different amounts of inventory to each client. Our work also considers competition between two networks. Yet, we find a simple and intuitive relationship between the inventories of the aggregate problem and the multiple client problem.

\subsection{Availability-Dependent Scatter Price}

Our analyses in Sections 3 and 4 assume that the scatter price depends on show performances but is independent of the availability of slots (recall that scatter price is based on per 30-second slot). In this sub-section, we relax this assumption and allow the scatter price to depend on the availability of advertising slots (or simply the capacity) as well as on the show performances. The capacity available for network $i, i$ $=1,2$, during the scatter market is given by: 


$$
C_{i}=\left\{\begin{array}{lc}
N_{i}-X_{i} / v_{i}, & \varepsilon_{i} \geq v_{i} \\
N_{i}-X_{i} / \varepsilon_{i}, & X_{i} / N_{i} \leq \varepsilon_{i} \leq v_{i} \\
0, & \varepsilon_{i} \leq X_{i} / N_{i} .
\end{array}\right.
$$

The first part of equation (11) represents the scenario where the committed number of slots committed in upfront is sufficient to meet the upfront rating points commitments as well; while the second part represents the opposite scenario where a network needs make good allocation of slots to deliver on the upfront rating points commitments. The third part of (11) represents the situation where the network pays a penalty as it is unable to deliver on the upfront rating points commitments. We consider two demand functions for the scatter market:

$$
\begin{aligned}
& p_{s i}=\varepsilon_{i}\left(\theta_{c i}-\eta_{i} C_{i}\right), \eta_{i}>0, \text { and } \\
& p_{s i}=\theta_{c i} \varepsilon_{i} C_{i}^{-\eta_{i}}, \eta_{i}<1, \theta_{c i} \geq 0 .
\end{aligned}
$$

The demand functions in equations (12a) and (12b), respectively, are additive and multiplicative in capacity. The parameter $\eta_{i}$ captures the price elasticity of capacity. To avoid trivial solutions, we will assume the unit penalty $g_{i}>\theta_{c i}$ in demand function (12a). The resulting prices of both demand functions are increasing in show performances. In particular, (12b) is similar to the multiplicative model adopted by Araman and Popescu (2010).

\section{Proposition 8:}

(a) There exists a unique equilibrium to the Cournot game with capacity-dependent scatter price under both additive and multiplicative demand functions.

(b) The equilibrium upfront inventory of network $i, X_{i}^{*}$, is increasing in the parameter $\eta_{i}$ under both additive and multiplicative demand functions.

The existence of unique equilibrium assures us about the feasibility of the scenario being studied in this sub-section. In fact, it could be shown that the sensitivity results described in Section 4 of the paper continue to hold even under capacity-dependent scatter price. It could also be shown that the equilibrium expected profit is increasing in $v_{i}$, the negotiated expected rating point of a slot. This suggest that exaggerating show performances remains a good strategy for a network under many different scenarios. Proposition 8(b) indicates that as the elasticity parameter $\eta_{i}$ increase so does the equilibrium upfront inventory. As $\eta_{i}$ increases, the scatter price decreases, making the scatter market less attractive to a network. Upfront inventory, consequently, goes up. Numerical experiments similar to Numerical Study 1 and 2 confirm that equilibrium upfront guarantee, $X_{i}^{*}$, increases with the mean of show performances and decreases with the variance of show performances. These observations are similar to those from Figures 1 through 4 and are 
omitted for the reasons of brevity. Availability-dependent scatter price, thus, does not fundamentally change the dynamics of upfront inventory allocation problem.

\subsection{Information Asymmetry}

Our analyses in Sections 4 assumes that the each network knows the distribution of its rating point as well as that of its competitor's. It is a valid assumption when there are few new shows from either network during the broadcast year. However, when there are many new shows during the broadcast year, a network might have better information about its show performance than that of its competitor's. In this section, we extend our analysis to information asymmetry to model such scenarios. Cournot competition under incomplete information of production cost has been well-studied in economics literature (Basar and Ho 1974, Li 1985, Shapiro 1986, Vives 2002). The standard technique is to find Bayesian Nash equilibrium which requires that each network maximizes its profits in expectation, assuming the other network does the same.

We characterize network $i$ by its type, parameterized by $l_{i}(i=1,2)$. We assume $l_{i}$ to be the private information of network $i$. Network $j$ 's prior on $l_{i}(i, j=1,2 ; j \neq i)$ is drawn randomly from a continuous distribution with cumulative distribution function $F_{l}($.), which is common knowledge to both networks. We assume $F_{l}\left(\right.$.) to be continuously differentiable. Let $X_{j}\left(l_{j}\right)$ be network $j$ 's upfront inventory response to $X_{i}$ given its knowledge of $l_{j}$ and the common prior $F_{l}($.). Network $i$ 's expected profit, then, is given by:

$$
\begin{aligned}
& \underset{X_{i}}{\operatorname{Max}} \pi_{i}=\int\left(p_{i} X_{i}+E\left[\pi_{s i}\right]\right) d F_{l}\left(l_{j}\right), i, j=1,2 ; j \neq i, \text { where, } \\
& p_{i}=\alpha_{i}-\beta_{i} X_{i}-\gamma X_{j}\left(l_{j}\right) .
\end{aligned}
$$

Substituting (13b) into (13a) and taking derivatives, we get,

$$
\partial \pi_{i} / \partial X_{i}=\alpha_{i}-2 \beta_{i} X_{i}-\gamma \int X_{j}\left(l_{j}\right) d F_{l}\left(l_{j}\right)-\theta_{i}^{\prime}-\left(g_{i}-\theta_{i}\right) F_{i}\left(X_{i} / N_{i}\right)
$$

It is well known that in a Bayesian game with continuous strategy spaces and continuous types, if strategy sets and type sets are compact, payoff functions are continuous and concave in a player's own strategies, then a pure strategy Bayesian Nash equilibrium exists. Given our profit functions in (13a) and (13b), our assumptions about the finite support of the random variable representing show performances and the differentiability of the respective CDFs, and that the player types are drawn randomly from a continuous distribution, all of the conditions of existence of pure strategy Nash equilibrium are satisfied. Therefore, a pure strategy Bayesian Nash equilibrium exists under information asymmetry.

Following an approach similar to the proof of proposition 2(a), it is easy to verify that Proposition

2 still holds under the Bayesian equilibrium. This suggests that the sensitivity analyses described in Proposition 2 are robust and continue to hold under information asymmetry. 
Given the complexity of the problem, characterizing the equilibrium upfront inventory $X_{i}^{*}$ under information asymmetry for arbitrary general distributions of show performances and the common prior is rather difficult. Thus, to gain additional insights about the problem we make specific distributional assumptions. We assume $\varepsilon_{i}$, the random variable denoting the show performance, follows a uniform distribution over $\left[\mu-l_{i}, \mu+l_{i}\right], i=1,2$, where, $l_{i}$ is private information of network $i$, while $\mu$ is common knowledge. We further assume that the distribution of common prior of the player types also follows a uniform over $[0, d]$. As the following proposition states, it is, indeed, possible to show that under certain conditions, the equilibrium upfront inventory of each network is increasing in the parameter $d$.

Proposition 9: Assume that the two networks are symmetric in parameters and that the relation $v \geq \mu$ holds. Then if $\alpha-2 \beta \mu N-\frac{g}{2}-\frac{\theta\left(\mu_{i}+l_{i}\right)}{2 v} \leq 0, i=1,2$, then the equilibrium upfront inventory of each network increases in the parameter $d$.

The parameter $d$ in our formulation is a measure of the uncertainty of the show performance as well as the accuracy of the prior. A higher value of the parameter $d$ indicates that network $i$ conjectures a high variability in the show performance of network $j$, and hence, a lower upfront inventory choice from network $j$. Thus, its own equilibrium upfront inventory choice increases. The findings of Proposition 9 is similar to the findings from Proposition 4, where we showed that under full information, a network's equilibrium choice increases as the variability of the show performance of its competitor increases. The key difference between the two results is as follows. Under full information, the uncertainty that network $i$ associates with network $j$ is solely due to the uncertainty of show performance. Under information asymmetry, this uncertainty arises because of combination of uncertainties of show performance and the accuracy of the prior. Thus, when the prior information is perfect, Proposition 9 reduces to Proposition 4.

\section{SUMMARY AND CONCLUSION}

Allocating the inventory of rating points between the upfront and the scatter markets is one of the most strategic decisions a television network makes. In this paper, we develop a game theoretic model to accomplish this. Our work considers two television networks whose rating points inventories could be substitutes; and explicitly accounts for the randomness of show performances as well as the scatter market prices. We have established unique Nash equilibrium of the Cournot game where each network chooses the amount of rating points inventory. We show that upfront inventories of two networks are strategic substitutes and describe the sensitivity of the equilibrium outcome with respect to various problem parameters. We show that choosing quantity over price is a dominant strategy for a network irrespective of the choice of its competitor. We compare our competitive model with a centralized one and describe the sensitivity of the equilibrium profit differential between the centralized and the competitive model with 
respect to the problem parameters. Finally, we consider three extensions to our basic model to include client-level allocation of the upfront inventory, availability-and-performance-dependent scatter market pricing, and information asymmetry.

Our work adds to the growing stream of literature studying various operational/supply chain uncertainties and makes the following important contributions to the operations management literature. First, to the best of our knowledge, ours is the first to study the media revenue management problem using a competitive setting. In addition, we are aware of only two papers (Bollapragada and Mallik, 2008; Araman and Popescu, 2010) that study the single-firm media revenue management problem using the upfront and the scatter markets. Our work can be viewed as a competitive generalization of these two papers that allows us to model the strategic interaction between the two television networks. Second, while there is little transparency in practice to show that the television networks engage in quantity competition, we have shown that the dominant strategy of a television network is to choose quantity (i.e., rating points) irrespective of the action of its competitor. Our work, thus, provides a theoretical validation for making quantity the key decision variable in media revenue management problems. Such theoretical validation is not provided by earlier works. Third, our work shows that the negotiated expected rating points $v$ plays an important role in the upfront inventory decisions of a network. We show that a network has incentive to deliberately inflate the show performances as its expected revenue is increasing in $v$. Furthermore, as $v$ increases, the deviation between expected revenues from the centralized and the competitive model decreases. Fourth, while extending our analyses to multiple client upfront planning, we show that there is a simple additive relationship of the equilibrium upfront inventories between the multiple client inventory planning and the aggregate inventory planning. Finally, our work has the following prescriptive and descriptive implications for a practicing manager.

- Proposition 5 of our paper indicates that a television network is better off deciding the inventory of rating points during the upfront market rather than deciding the price. This result continues to hold irrespective of the decision of its competitor, or the show performances of either network. The current practice in the industry shows that consistent decision rules (on price vs. quantity) are seldom followed by the decision makers. Media reports also suggest that managers tend to negotiate price first when the shows are expected to perform strongly. Our work, thus, provides an important prescriptive guideline for the practitioners.

- Proposition 2(b) of our papers shows that selling more in a strong upfront is, indeed, an optimal strategy for a network. This practice is widely followed by both broadcast and cable networks. Our work, thus provides an explanation for the observed practices. Proposition 4 also provides an important qualitative guiding principle to a manager: a network with highly uncertain show performances (for example, when there are many new shows) should sell less inventory during the upfront. 
- Lemma 1 is also useful to a practitioner as it provides insights about how to react to a competitor's inventory decisions and tells a manager that the upfront inventories of the two networks are strategic substitutes. Finally, the sensitivity analyses presented in the paper are important guiding tools for a practitioner as well.

- Even when a television network charges different upfront prices to each customer, per Proposition 7, it can still obtain the optimal aggregate upfront inventory simply by adding the optimal inventory for each customer. This simple and intuitive insight, we believe, is rather valuable to a practitioner planning the upfront strategy for a television network.

- We show in Section 5.3 that presence of information asymmetry does not alter our sensitivity analyses results. This assures a practitioner about the robustness of our results.

Like any other work in operations management, our work is not free from assumptions. Ours is a single period model. Extending our results to multiple periods will allow us to capture the strategic interactions between the networks over a period of time. It will also allow us to capture such effects as a strong scatter price during a year results in a higher upfront demand during the following year and vice versa, and the effect of running a show over multiple time periods. Our work assumes risk neutrality where the television networks are expected revenue maximizers. While this assumption is standard in operations management literature, it will be useful to extend our analyses to risk-averse utility functions of the networks and/or to the framework adopted by Bollapragada and Mallik (2008) where a network maximizes its expected revenue subject to a chance constraint that the revenue exceeds a target value with a given probability. Our model assumes all ad slots of a network are homogenous in the sense that they capture identical rating points. Relaxing this assumption is also a valuable extension of our model.

\section{REFERENCES}

Alaei, R., F. Ghassemi-Tari. 2011. Development of a Genetic Algorithm for Advertising Time Allocation Problems. Journal of Industrial and Systems Engineering. 4, 245-255.

Araman V.F., I. Popescu. 2010. Media Revenue Management with Audience Uncertainty; Balancing Upfront and Spot Market Sales, Manufacturing \& Service Operations Management, 12(2), 190-212.

Barsar, T., Y. Ho, 1974. Informational Properties of the Nash Solutions of Two Stochastic Nonzero-Sum Games. Journal of Economic Theory, 7, 370-387.

Bitran, G., R. Caldentey. 2003. An Overview of Pricing Models for Revenue Management. Manufacturing \& Service Operations Management. 5(3), 203-230.

Bollapragada, S., H.Cheng, M. Phillips, M. Scholes, T. Gibbs, M. Humphreville. 2002. NBC's Optimization Systems Increase its Revenues and Productivity. Interfaces. 32(1).

Bollapragada, S., M.R. Bussieck, S. Mallik. 2004. Scheduling Commercial Videotapes in Broadcast Television. Operations Research, 52(5), 679-689. 
Bollapragada, S., M. Garbiras. 2004. Scheduling Commercials on Broadcast Television. Operations Research, 52(3), 337-345.

Bollapragada S., S. Mallik. 2008. Managing On-air Ad Inventory in Broadcast Television, IIE Transactions 40, 1107-1123.

Cachon G.P., S. Netessine. 2004. Game Theory in Supply Chain Analysis. In book: Handbook of quantitative supply chain analysis: modeling in the e-business era, Publisher: Kluwer, Editors: SimchiLevi, David, David Wu, Max Shen, pp.13 - 59.

Cachon G.P., P. Feldman. 2017. Is Advance Selling Desirable with Competition? Marketing Science, 36(2), 214-231.

Cheng L. (1985), Comparing Bertrand and Cournot Equilibria: A Geometric Approach. Rand Journal of Economics 16(1), 146-151.

Chintapalli, P., S.M. Disney, C.S. Tang. 2017. Coordinating Supply Chains via Advance-Order Discounts, Minimum Order Quantities, and Delegations. Production and Operations Management. Forthcoming.

Chunovic, L. 2003. Upfront a record 9.3 Bil. Television Week. Vol. 20(21), p. 25, May 26, 2003.

Collins, S. 2014. Broadcast networks encouraged. Source: http://www.latimes.com/entertainment/tv/la-etst-tv-winners-losers-20141013-story.html, retrieved on December 26, 2016

Degraba, P. 1995. Buying frenzies and seller-induced excess demand. RAND Journal of Economics. 26(2), 331-342.

Eliashberg, J., S. Swami, C.B. Weinberg, B. Wierenga. 2001. Implementing and Evaluating SilverScreener: A Marketing Management Support System for Movie Exhibitors. Interfaces, 31(3), S108-S127.

Flint, J., S. Vranica. 2016. TV Ad Spending Shows Signs of Revival. The Wall Street Journal. April 18, 2016, p. B1.

Friedman, W. 2015. Broadcast, Cable Upfront Spending Drops Again. Source: http://www.mediapost.com/publications/article/257973/broadcast-cable-upfront-spending-dropsagain.html, Retrieved on July 25, 2016.

Gallego, G., O. Sahin. 2010. Revenue Management with Partially Refundable Fares. Operations Research. 58(4), 817-833.

Garcia-Villoria, A., S. Salhi. 2015. Scheduling commercial advertisements for television. International Journal of Production Research. 53(4), 1198-1215.

Gaur, D.R., R. Krishnamurti, R. Kohli. 2009. Conflict Resolution in the Scheduling of Television Commercials. Operations Research, 57(5), 804-807.

Gerchak, Y., D. Mossman. 1992. Technical notes: On the effect of demand randomness on inventories and costs. Operations Research, 40, 1098-1105.

Ghassemi-Tari, F., R. Alaei. 2013. Scheduling TV commercials using genetic algorithms. International Journal of Production Research. 54(18), 5499-5521.

Goodhardt, G.J., A.S.C. Ehrenberg, M.A. Collins. 1975. The Television Audience: Patterns of Viewing. Saxon House, Westmead, England.

Grosfeld-Nir, A., Y. Gerchak. 2004. Multiple lot sizing in production to order with random yields: Review of recent advances. Annals of Operations Research, 126, 43-69. 
Guerriero, F., G. Miglionico, F. Olivito. 2016. Managing TV commercials inventory in the Italian advertising market. International Journal of Production Research. 54(18), 5499-5521.

Guo, L. 2009. Service Cancellations and Competitive Refund Policy. Marketing Science. 28(5), 901-907.

Headen, R.S., J.E. Klompmaker, R.T. Rust. 1979. The Duplication of Viewing Law and Television Media Scheduling Evaluation. Journal of Marketing Research. 16, 333-340.

Henry, M.D., H.J. Rinne. 1984. Predicting Program Shares in New Time Slots. Journal of Advertising Research. 24(2), 9-17.

Jones, J., G.J. Koehler. 2002. Combinatorial Auctions using Rule-Based Bids. Decision Support Systems. 34(1), 59-74.

Kleindorfer, P., D.J. Wu. 2003. Integrating long-term and short-term contracting via B2B exchanges for capital-intensive industries. Management Science, 49(11), 1597-1615.

Lafayette, J., W. Friedman. 2004. CBS and ABC Holdouts in Slow Upfront. Television Week. 23(24), 0-1.

Li, L., 1985. Cournot Oligopoly with Information Sharing. Rand Journal of Economics, vol. 16, No. 4, 521-536.

Li, Q., D. Atkins. 2002. Coordinating replenishment and pricing in a firm. Manufacturing \& Service Operations Management, 4, 241-257.

McCardle, K.F., K. Rajaram, C.S. Tang. 2004. Advance Booking Discount Programs under retail Competition. Management Science. 50(5), 701-708.

Moulin, H. (1986). Game Theory for the Social Science. New York University Express.

Philips R., G. Young. 2012. Television Advertisement Pricing in the US. In The Oxford Handbook of Pricing Management. Eds. Özalp Özer, Robert Phillips. 181-198. ISBN 978-0-19-954317-5.

Reddy, S.K., J.E. Aronson, A. Stam. 1998. SPOT: Scheduling Programs Optimally for Television. Management Science. 44(1), 83-102.

Rust, R.T., N.V. Echambadi. 1989. Scheduling Network Television Programs: A Heuristic Audience Flow Approach to Maximizing Audience Share. Journal of Advertising. 18(2), 11-18.

Shapiro, C., 1986. Exchange of Cost Information in Oligopoly. Review of Economic Studies, LIII, 433446.

Shields, M. 2016. As TV Market Rebounds, Programmatic Takes a Back Seat. The Wall Street Journal. July 25, 2016.

Shugan, S.M., J. Xie. 2005. Advance-selling as a competitive marketing tool. International Journal of Research in Marketing. 22(3), 351-373.

Singh N., X. Vives (1984), Price and Quantity Competition in a Differentiated Duopoly, Rand Journal of Economics 15(4), 546-554.

Somlo, B., K. Rajaram, R. Ahmadi. 2011. Distribution Planning to Optimize Profits in the Motion Picture Industry. Production and Operations Management, 20(4), 618-636.

Song, Y., S. Ray, S. Li. 2008. Structural properties of buy-back contracts for price-setting newsvendors. Manufacturing \& Service Operations Management, 10, 1-18.

Steinberg, B. 2005. Not Desperate for Ad Commitments; Thanks to Strong Ratings And Subdued Strategy, ABC Wraps Up Its 'Upfront' Sale. The Wall Street Journal. June 1, 2005. 
Steinberg, B. 2016a. Upfront 2016: TV Networks May Gain Upper Hand For First Time In Years. March 14, 2016. Source: http://variety.com/2016/tv/news/2016-tv-upfront-tv-networks-ad-rates-tv-advertising1201729219/. Retrieved on January 3, 2017.

Steinberg, B. 2016b. TV’s 'Upfront’ Ad-Sales Talks Start to Heat Up. June 15, 2016. Source: http://variety.com/2016/tv/news/2016-tv-upfront-advertising-nbcuniversal-cbs-1201796454/. Retrieved on January 3, 2017.

Steinberg, B. 2016c. Influential Media Buyer GroupM Seeks Big Changes in TV Measurement for 2017 Upfront. http://variety.com/2016/tv/news/tv-advertising-upfront-lyle-schwartz-groupm-1201928716/. Retrieved on May 16, 2017.

Steinberg, B. 2017. CBS Releases ‘Total Content' Viewership Measures as Upfront Market Looms. Source: http://variety.com/2017/tv/news/cbs-total-content-ratings-nielsen-upfront-2017-advertising1202002685/. Retrieved on May 16, 2017

Swami, S., J. Eliashberg, C.B. Weinberg. 1999. SilverScreener: A Modeling Approach to Movie Screens Management. Marketing Science, 18(3), 352-372.

Talluri, T.T., G.J. van Ryzin. 2004. The theory and Practice of Revenue Management. Kluwer Academic Publishers, ISBN 1-4020-7701-7.

Tang, C.S., K. Rajaram, A. Alptekinoglu, J. Ou. 2004. The benefits of Advance Booking Discount Programs: Model and Analysis. Management Science. 50(4), 465-478.

Tsay A.A., N. Agrawal. 2000. Channel Dynamics Under Price and Service Competition. Manufacturing \& Service Operations Management, 2(4), 372-391.

Vives, X., 2002. Private Information, Strategic Behavior, and Efficiency in Cournot Markets. Rand Journal of Economics, vol. 33, No.3, 361-376.

Vranica, S. 2006. Advertisers Turn to eBay to Buy TV Time. The Wall Street Journal. August 4, 2006, p. A11.

Webster, J.G. 1985. Program Audience Duplication: A Study of Television Inheritance Effects. Journal of Broadcasting \& Electronic Media. 29(2), 121-133.

Weng, K., M. Parler. 1999. Integrating Early Sales with Production Decisions: Analysis and Insights. IIE Transactions, 31(11), 1051-1060.

Wu, D.J., P. Kleindorfer. 2005. Competitive options, supply contracts and electronic markets. Management Science, 51(3), 452-466.

Xie, J., S.M. Shugan. 2001. Electronic Tickets, Smart Cards, and Online Prepayments: When and How to Advance Sell. Marketing Science, 20(3), 219-243.

Xie, J., E. Gerstner. 2007. Service Escape: Profiting from Customer Cancellations. Marketing Science. 26(1), 18-30.

Yano, C. A., H. L. Lee. 1995. Lot sizing with random yields: A review. Operations Research, 43(2), 311334.

Yu, M., R. Kapuscinski, H. Ahn. 2015. Advance Selling: Effects of Interdependent Consumer Valuations and Seller’s Capacity. Management Science. 61(9), 2100-2117. 


\title{
ONLINE APPENDIX
}

\section{MANAGING TELEVISION COMMERCIAL INVENTORY UNDER COMPETITION: AN EQUILIBRIUM ANALYSIS}

\author{
Qin Geng \\ College of Business, Kutztown University of Pennsylvania, Kutztown, PA 19530, geng@kutztown.edu \\ Suman Mallik \\ School of Business, University of Kansas, Lawrence, KS 66045, suman@ku.edu
}

\section{PROOFS OF ALL RESULTS}

\section{Proof of Lemma 1:}

From equation (3), $\frac{\partial^{2} \pi_{i}}{\partial X_{i}^{2}}=-2 \beta_{i}-\left[\frac{\left(g_{i}-\theta_{i}\right)}{N_{i}}\right] f_{i}\left(\frac{X_{i}}{N_{i}}\right) \leq 0 ; \frac{\partial^{2} \pi_{i}}{\partial X_{i} X_{j}}=-\gamma \leq 0$.

Therefore, $\frac{d X_{i}\left(X_{j}\right)}{d X_{j}}=-\left(\frac{\partial^{2} \pi_{i}}{\partial X_{i} \partial X_{j}}\right) /\left(\frac{\partial^{2} \pi_{i}}{\partial X_{i}^{2}}\right) \leq 0$.

\section{Proof of Proposition 1:}

$\left|\frac{\partial^{2} \pi_{i}}{\partial X_{i} \partial X_{j}}+\frac{\partial^{2} \pi_{j}}{\partial X_{j} \partial X_{i}}\right|=2 \gamma<2 \beta_{i} \beta_{j}<\sqrt{\left|\frac{\partial^{2} \pi_{i}}{\partial X_{i}^{2}} \frac{\partial^{2} \pi_{j}}{\partial X_{j}^{2}}\right|}$.

Therefore there exists a unique Nash equilibrium (see Moulin 1986).

Proof of Proposition 2(a):

Let $L_{1}=\frac{\partial \pi_{1}}{\partial X_{1}}=\alpha_{1}-2 \beta_{1} X_{1}-\gamma X_{2}-\theta_{1}-\left(\frac{\theta_{1}}{v_{1}}\right) \int_{v_{1}}^{B_{1}}\left(1-F_{1}\left(\varepsilon_{1}\right) d \varepsilon_{1}-\left(g_{1}-\theta_{1}\right) F_{1}\left(X_{1} / N_{1}\right)\right.$.

By Theorem 9 in Cachon and Netessine (2004),

$\frac{d X_{1}}{d \alpha_{1}}=-\frac{\frac{\partial^{2} \pi_{1} \partial^{2} \pi_{2}}{\partial X_{1} \partial \alpha_{1} \partial X_{2}^{2}}-\frac{\partial^{2} \pi_{2}}{\partial X_{1} \partial X_{2} \partial X_{2} \partial \alpha_{1}}}{|H|}$, where,

$|\mathrm{H}|=\left|\begin{array}{cc}\frac{\partial^{2} \pi_{1}}{\partial X_{1}^{2}} & \frac{\partial^{2} \pi_{1}}{\partial X_{1} \partial X_{2}} \\ \frac{\partial^{2} \pi_{1}}{\partial X_{1} \partial X_{2}} & \frac{\partial^{2} \pi_{2}}{\partial X_{2}^{2}}\end{array}\right|>0$, and $\frac{\partial^{2} \pi_{2}}{\partial X_{2} \partial \alpha_{1}}=0, \frac{\partial^{2} \pi_{2}}{\partial X_{2}^{2}} \leq 0$.

Therefore, sign of $\left(\frac{d X_{1}}{d \alpha_{1}}\right)=\operatorname{sign}$ of $\left(\frac{\partial^{2} \pi_{1}}{\partial X_{1} \partial \alpha_{1}}\right)=\operatorname{sign}$ of $\left(\frac{\partial L_{1}}{\partial \alpha_{1}}\right) \geq 0$.

The proofs for sensitivity results with respect to the parameters $\beta, g, v$, and $N$ are similar, and hence are omitted. We provide the proof of sensitivity result with respect to $\theta_{i}$ next. 


$$
\begin{aligned}
& \frac{\partial L_{1}}{\partial \theta_{1}}=-\left(1-F_{1}\left(X_{1} / N_{1}\right)-\left(\frac{1}{v_{1}}\right) \int_{v_{1}}^{B_{1}}\left(1-F_{1}\left(\varepsilon_{1}\right) d \varepsilon_{1} \leq 0 .\right.\right. \\
& \frac{\partial L_{1}}{\partial v_{1}}=-\theta_{1}\left[-\int_{v_{1}}^{B_{1}}\left(1-F_{1}\left(\varepsilon_{1}\right)\right) d \varepsilon_{1}-\left(1-F_{1}\left(v_{1}\right)\right) v_{1}\right] / v_{1}^{2} \geq 0 .
\end{aligned}
$$

\section{Proof of Proposition 2(b):}

For the symmetric game, the FOC of the expected profit function in equation (3) becomes:

$\alpha-(2 \beta+\gamma) X-\theta^{\prime}-(g-\theta) F\left(\frac{X}{N}\right)=0$.

The LHS of the above expression is decreasing in $\gamma$. Therefore, $\partial X^{*} / \partial \gamma<0$.

Or sign of $\left(\frac{d X}{d \gamma}\right)=-\operatorname{sign}$ of $\left(\frac{\partial^{2} \pi_{1}}{\partial X_{1} \partial \gamma} \frac{\partial^{2} \pi_{2}}{\partial X_{2}^{2}}-\frac{\partial^{2} \pi_{2}}{\partial X_{1} \partial X_{2}} \frac{\partial^{2} \pi_{2}}{\partial X_{2} \partial \gamma}\right)=\operatorname{sign}$ of $\left((2 \beta-r) X+(g-\theta) f\left(\frac{X}{N}\right) / N\right) \geq 0$.

The proofs for other results are similar.

\section{Proof of Proposition 3:}

$\frac{d \pi_{i}\left(X_{i}^{*}, X_{j}^{*}\right)}{d v i}=\frac{\partial \pi_{i}\left(X_{i}^{*}, X_{j}^{*}\right)}{\partial X i} \frac{\partial X_{i}^{*}}{\partial v_{i}}+\frac{\partial \pi_{i}\left(X_{i}^{*}, X_{j}^{*}\right)}{\partial X j} \frac{\partial X_{j}^{*}}{\partial v_{i}}+\frac{\partial \pi_{i}\left(X_{i}^{*}, X_{j}^{*}\right)}{\partial v i}$, where,

$\frac{\partial \pi_{i}\left(X_{i}^{*}, X_{j}^{*}\right)}{\partial v i}=\int_{v_{i}}^{B_{i}} X_{i} / v_{i}^{2} \theta_{i} \varepsilon_{i} f_{i}\left(\varepsilon_{i},\right) \mathrm{d} \varepsilon_{i} \geq 0$,

$\frac{\partial \pi_{i}\left(X_{i}^{*}, X_{j}^{*}\right)}{\partial X i}=0$ at $\left(X_{i}^{*}, X_{j}^{*}\right)$,

$\frac{\partial \pi_{i}\left(X_{i}^{*}, X_{j}^{*}\right)}{\partial X j}=-\gamma X_{i} \leq 0$, and, $\frac{\partial X_{j}^{*}}{\partial v_{i}} \leq 0$.

By Proposition 2, therefore, $\frac{d \pi_{i}\left(X_{i}^{*}, X_{j}^{*}\right)}{d v i} \geq 0$.

Similarly, $d \pi_{i}\left(X_{i}^{*}, X_{j}^{*}\right) / d v_{j}=\frac{\partial \pi_{i}\left(X_{i}^{*}, X_{j}^{*}\right)}{\partial X i} \frac{\partial X_{i}^{*}}{\partial v_{j}}+\frac{\partial \pi_{i}\left(X_{i}^{*}, X_{j}^{*}\right)}{\partial X j} \frac{\partial X_{j}^{*}}{\partial v_{j}}+\frac{\partial \pi_{i}\left(X_{i}^{*}, X_{j}^{*}\right)}{\partial v j} \leq 0$.

\section{Proof of Proposition 4:}

$$
\begin{aligned}
\frac{\partial \pi_{i}}{\partial X_{i}}= & \alpha_{i}-2 \beta_{i} X_{i}-\gamma X_{j}-\int_{v_{i}}^{B_{i}} \frac{\theta_{i}}{v_{i}}\left(\left(1-\tau_{i}\right) \mu_{i}+\tau_{i} \varepsilon_{i}\right) f_{i}\left(\varepsilon_{i}\right) d \varepsilon_{i}-\theta_{i} F_{i}\left(v_{i}\right)-\left(g_{i}-\theta_{i}\right) F_{i}\left(\frac{X_{i}}{N_{i}}\right) \\
& -\left(g_{i}-\theta_{i}\right)\left(1-\tau_{i}\right)\left(\frac{X_{i}}{N_{i}}-\mu_{i}\right) f_{i}\left(\frac{X_{i}}{N_{i}}\right)=0 .
\end{aligned}
$$

It can be shown that sign of $\left(\partial X_{i} / \partial \tau_{i}\right)=\operatorname{sign}$ of $\left(\partial^{2} \pi_{i} / \partial X_{i} \partial \tau_{i}\right)$, where,

$$
\partial^{2} \pi_{i} / \partial X_{i} \partial \tau_{i}=\int_{v_{i}}^{B_{i}} \frac{\theta_{i}}{v_{i}}\left(\mu_{i}-\varepsilon_{i}\right) f_{i}\left(\varepsilon_{i}\right) d \varepsilon_{i}-\left(g_{i}-\theta_{i}\right)\left(\mu_{i}-\frac{X_{i}}{N_{i}}\right) f_{i}\left(\frac{X_{i}}{N_{i}}\right),
$$

which is negative when $v_{i} \geq \mu_{i} \geq X_{i} / N_{i}$.

Next, we find a sufficient condition for $\mu_{i} \geq X_{i} / N_{i}$, or equivalently, $X_{i} \leq N_{i} \mu_{i}$. Note that:

$\frac{\partial \pi_{i}}{\partial X_{i}}<\alpha_{i}-2 \beta_{i} X_{i}-\tau_{i}\left(\frac{\theta_{i}}{v_{i}} \int_{v_{i}}^{B_{i}} \varepsilon_{i} f_{i}\left(\varepsilon_{i}\right)+\theta_{i} F_{i}\left(v_{i}\right)\right)-\left(g_{i}-\theta_{i}\right) F_{i}\left(\frac{X_{i}}{N_{i}}\right)$.

The RHS of the above inequality decreases in $X_{i}$.

If at $X_{i}=N_{i} \mu_{i}, \alpha_{i}-2 \beta_{i} N_{i} \mu_{i}-\tau_{i}\left(\frac{\theta_{i}}{v_{i}} \int_{v_{i}}^{B_{i}} \varepsilon_{i} f_{i}\left(\varepsilon_{i}\right)+\theta_{i} F_{i}\left(v_{i}\right)\right)-\left(g_{i}-\theta_{i}\right) F_{i}\left(\mu_{i}\right) \leq 0$,

then $X_{i}$ that satisfies $\partial \pi_{i} / \partial X_{i}=0$ must be less than $N_{i} \mu_{i}$ as well.

It can be shown that sign of $\left(\partial X_{i} / \partial \tau_{j}\right)=-\operatorname{sign}$ of $\left(\partial^{2} \pi_{j} / \partial X_{j} \partial \tau_{j}\right) \geq 0$.

\section{Proof of Proposition 5(a):}


The proof of Proposition 5 is rather tedious. As a result, we divide the proofs into four lemmas, labeled Lemma A1 through Lemma A4. The proof of this proposition follows directly from the proofs of these four lemmas. To facilitate the exposition, we will use superscripts to denote a model (Q, P, B, and C) and subscripts to denote a television network (1 and 2). For example, $X_{1}^{P}$ is the quantity of network 1 in model $\mathrm{P}$, and $X_{2}^{P}$ is the quantity of network 2 in model $\mathrm{P}$.

Lemma A1. $X_{2}^{C} \leq X_{2}^{P}$.

Proof: From FOC of model C,

$$
\begin{aligned}
& \alpha_{1}-2 \beta_{1} X_{1}-\gamma X_{2}-\theta_{1}^{\prime}-\left(g_{1}-\theta_{1}\right) F_{1}\left(X_{1} / N_{1}\right)=0 \\
& \alpha_{2}-2 \beta_{2} X_{2}-\gamma X_{1}-\theta_{2}^{\prime}-\left(g_{2}-\theta_{2}\right) F_{2}\left(X_{2} / N_{2}\right)=0 .
\end{aligned}
$$

Solving for $X_{1}$ From (A2), we get, $X_{1}=\left[\alpha_{2}-2 \beta_{2} X_{2}-\theta_{2}^{\prime}-\left(g_{2}-\theta_{2}\right) F_{2}\left(\frac{X_{2}}{N_{2}}\right)\right] / \gamma$.

Plugging the above expression of $X_{1}$ into (A1), we get

$$
\begin{aligned}
\alpha_{1}-\frac{2 \beta_{1}}{\gamma}\left[\alpha_{2}-2 \beta_{2} X_{2}-\theta_{2}^{\prime}-\right. & \left.\left(g_{2}-\theta_{2}\right) F_{2}\left(\frac{X_{2}}{N_{2}}\right)\right]-\gamma X_{2}-\theta_{1}^{\prime} \\
& -\left(g_{1}-\theta_{1}\right) F_{1}\left(\frac{\alpha_{2}-2 \beta_{2} X_{2}-\theta_{2}^{\prime}-\left(g_{2}-\theta_{2}\right) F_{2}\left(\frac{X_{2}}{N_{2}}\right)}{\gamma N_{1}}\right)=0 .
\end{aligned}
$$

The LHS of (A3) is increasing in $X_{2}$ since $\left(4 \beta_{1} \beta_{2}-\gamma\right)>0$.

The FOC of model $\mathrm{P}$, given $X_{2}$, is:

$$
\partial \pi_{1} / \partial p_{1}=\frac{\alpha_{1}-\gamma X_{2}-2 p_{1}}{\beta_{1}}+\theta_{1}^{\prime}+\frac{\left(g_{1}-\theta_{1}\right) F_{1}\left(\frac{X_{1}}{N_{1}}\right)}{\beta_{1}}=0
$$

Substituting $p_{1}=\alpha_{1}-\beta_{1} X_{1}-\gamma X_{2}$, from equation (2a), into the above expression, we get, $\frac{\partial \pi_{1}}{\partial p_{1}}=\alpha_{1}-2 \beta_{1} X_{1}-\gamma X_{2}-\theta_{1}^{\prime}-\left(g_{1}-\theta_{1}\right) F_{1}\left(\frac{X_{1}}{N_{1}}\right)=0$, which identical to equation (A1).

On the other hand, given $p_{1}, \partial \pi_{2} / \partial X_{2}=\frac{a_{2}+c p_{1}-2 X_{2}}{b_{2}}-\theta_{2}^{\prime}-\left(g_{2}-\theta_{2}\right) F_{2}\left(\frac{X_{2}}{N_{2}}\right)=0$.

Using equation 2(a), and $a_{i}=\left(\alpha_{i} \beta_{j}-\alpha_{j} \gamma\right) /\left(\beta_{i} \beta_{j}-\gamma^{2}\right), b_{i}=\beta_{i} /\left(\beta_{i} \beta_{j}-\gamma^{2}\right)$, and $c=\gamma /\left(\beta_{i} \beta_{j}-\gamma^{2}\right)$, the above expression of $\frac{\partial \pi_{2}}{\partial X_{2}}$ can be written as:

$\frac{\partial \pi_{2}}{\partial X_{2}}=\alpha_{2}-\left(2 \beta_{2}-\frac{\gamma^{2}}{\beta_{1}}\right) X_{2}-\gamma X_{1}-\theta_{2}^{\prime}-\left(g_{2}-\theta_{2}\right) F_{2}\left(\frac{X_{2}}{N_{2}}\right)=0$.

Solving for $X_{1}$ from the above equation, we get, $X_{1}=\frac{\alpha_{2}-\left(2 \beta_{2}-\frac{r^{2}}{\beta_{1}}\right) X_{2}-\theta_{2}^{\prime}-\left(g_{2}-\theta_{2}\right) F_{2}\left(\frac{X_{2}}{N_{2}}\right)}{\gamma}$.

Hence $\partial \pi_{1} / \partial p_{1}=0$ can be re-written as

$$
\begin{gathered}
\alpha_{1}-\frac{2 \beta_{1}}{\gamma}\left(\alpha_{2}-\left(2 \beta_{2}-\frac{\gamma^{2}}{\beta_{1}}\right) X_{2}-\theta_{2}^{\prime}-\left(g_{2}-\theta_{2}\right) F_{2}\left(\frac{X_{2}}{N_{2}}\right)\right)-\gamma X_{2}-\theta_{1}^{\prime} \\
-\left(g_{1}-\theta_{1}\right) F_{1}\left(\frac{\alpha_{2}-\left(2 \beta_{2}-\frac{\gamma^{2}}{\beta_{1}}\right) X_{2}-\theta_{2}^{\prime}-\left(g_{2}-\theta_{2}\right) F_{2}\left(\frac{X_{2}}{N_{2}}\right)}{\gamma N_{1}}\right)=0,
\end{gathered}
$$

which is increasing in $X_{2}$ since $\left(4 \beta_{1} \beta_{2}-\gamma-2 \gamma^{2}\right)>0$.

$X_{2}^{C}$ is, therefore, the solution to (A3) and that $X_{2}^{P}$ is the solution to (A4). Compare (A3) and (A4), we get $X_{2}^{C} \leq X_{2}^{P}$.

Lemma A2. Setting quantity dominates setting price given the other firm sets quantity, i.e., $\pi_{1}^{C} \geq \pi_{1}^{P}$.

Proof: $\pi_{1}^{p}=\pi_{1}\left(X_{1}^{p}, X_{2}^{p}\right) \leq \pi_{1}\left(X_{1}^{p}, X_{2}^{C}\right)$, as $X_{2}^{C} \leq X_{2}^{P}$ and $\left.\partial \pi_{1}\left(X_{1}, X_{2}\right) / \partial X_{2}=-\gamma \leq 0\right)$,

$$
\leq \pi_{1}\left(X_{1}^{C}, X_{2}^{C}\right) \text {, as } X_{1}^{C} \text { is the best response to } X_{2}^{C} \text {. }
$$

Lemma A3. $X_{1}^{Q} \geq X_{1}^{B} ; p_{1}^{Q} \geq p_{1}^{B} ; p_{2}^{Q} \geq p_{2}^{B}$.

Proof: From the FOCs of model B, $\partial \pi_{1} / \partial p_{1}=0$ and $\partial \pi_{2} / \partial p_{2}=0$, respectively, we get 
$a_{1}-2 b_{1} p_{1}+c p_{2}+b_{1}\left[\theta_{1}^{\prime}+\left(g_{1}-\theta_{1}\right) F_{1}\left(\left(a_{1}-b_{1} p_{1}+c p_{2}\right) / N_{1}\right)\right]=0$, and

$a_{2}-2 b_{2} p_{2}+c p_{1}+b_{2}\left[\theta_{2}^{\prime}+\left(g_{2}-\theta_{2}\right) F_{2}\left(\left(a_{2}-b_{2} p_{2}+c p_{1}\right) / N_{2}\right)\right]=0$.

Equation (A5) above can be re-written as:

$\alpha_{1}-\left(2 \beta_{1}-\frac{\gamma^{2}}{\beta_{2}}\right) X_{1}-\gamma X_{2}-\theta_{1}^{\prime}-\left(g_{1}-\theta_{1}\right) F_{1}\left(\frac{X_{1}}{N_{1}}\right)=0 ;$

while equation (A6) can be re-written as:

$\alpha_{2}-\left(2 \beta_{2}-\frac{\gamma^{2}}{\beta_{1}}\right) X_{2}-\gamma X_{1}-\theta_{2}^{\prime}-\left(g_{2}-\theta_{2}\right) F_{1}\left(\frac{X_{2}}{N_{2}}\right)=0$.

For model Q, the FOC $\partial \pi_{1} / \partial X_{1}=0$ is identical to equation (A7) above. The FOC $\partial \pi_{2} / \partial p_{2}=0$ can be written as:

$\alpha_{2}-2 \beta_{2} X_{2}-\gamma X_{1}-\theta_{2}^{\prime}-\left(g_{2}-\theta_{2}\right) F_{1}\left(\frac{X_{2}}{N_{2}}\right)=0$.

Let $\left(X_{1}^{B}, X_{2}^{B}\right)$ is the solution to equation (A7) and (A8), while $\left(X_{1}^{Q}, X_{2}^{Q}\right)$ is the solution to (A7) and (A9).

Following a logic similar to the proof of Lemma (A1) and assuming $\left(2 \beta_{2}-\gamma^{2} / \beta_{1}\right)\left(2 \beta_{1}-\gamma^{2} / \beta_{2}\right)-\gamma^{2} \geq 0$, we get $X_{1}^{Q} \geq X_{1}^{B}$. This proves the first part of Lemma A3.

Next, equation (A9) can be re-written as

$$
\frac{b_{1} b_{2}\left(a_{2}-2 b_{2} p_{2}+c p_{1}\right)+b_{2} c^{2} p_{2}}{b_{1} b_{2}-c^{2}}+b_{2}\left[\theta_{2}^{\prime}+\left(g_{2}-\theta_{2}\right) F_{2}\left(\left(a_{2}-b p_{2}+c p_{1}\right) / N_{2}\right)\right]=0 \text {. }
$$

Let $\left(p_{2}^{B}, p_{2}^{B}\right)$ is the solution to (A5) and (A6), while $\left(p_{1}^{Q}, p_{2}^{Q}\right)$ is the solution to (A5) and (A10). It is easy to verify that LHS of (A6) and (A10) are decreasing in $p_{2}$ for a given $p_{1}$. Therefore, by implicit function theory, $p_{2}^{Q}\left(p_{1}\right)$ and $p_{2}^{B}\left(p_{1}\right)$ increases in $p_{1}$. Moreover, LHS of (A10) is greater than LHS of (A6), therefore, $p_{2}^{Q}\left(p_{1}\right) \geq p_{2}^{B}\left(p_{1}\right)$ given the same $p_{1}$. Further, (A5) can be re-written as

$$
X_{1}-b_{1} p_{1}+b_{1}\left(\theta_{1}^{\prime}+\left(g_{1}-\theta_{1}\right) F_{1}\left(\frac{X_{1}}{N_{1}}\right)\right)=0 \text {. }
$$

Since $X_{1}^{Q} \geq X_{1}^{B}$, and LHS of (A11) increases in $X_{1}$ and decreases in $p_{1}, p_{1}^{Q} \geq p_{1}^{B}$.

Therefore, $p_{2}^{Q}=p_{2}^{Q}\left(p_{1}^{Q}\right) \geq p_{2}^{Q}\left(p_{1}^{Q}\right) \geq p_{2}^{B}\left(p_{1}^{Q}\right) \geq p_{2}^{B}\left(p_{1}^{B}\right)=p_{2}^{B}$

Lemma A4. Setting quantity dominates setting price given the other firm sets price, i.e., $\pi_{1}^{Q} \geq \pi_{1}^{B}$.

Proof: $\pi_{1}^{Q}=\pi_{1}\left(X_{1}^{Q}, X_{2}^{Q}\right)=\pi_{1}\left(p_{1}^{Q}, p_{2}^{Q}\right) \geq \pi_{1}\left(p_{1}^{B}, p_{2}^{Q}\right) \geq \pi_{1}\left(p_{1}^{B}, p_{2}^{B}\right)=\pi_{1}^{B}$

Note that $\pi_{1}\left(p_{1}^{Q}, p_{2}^{Q}\right) \geq \pi_{1}\left(p_{1}^{B}, p_{2}^{Q}\right)$. Given $p_{2}^{Q}$, network 1 's best quantity response, when written in $p$ -

space, is the same as the best price response function in model B, i.e, $p_{1}^{Q}$ is the best price response to $p_{2}^{Q}$. Note also that $\pi_{1}\left(p_{1}^{B}, p_{2}^{Q}\right) \geq \pi_{1}\left(p_{1}^{B}, p_{2}^{B}\right)$ as $\partial \pi_{1}\left(p_{1}, p_{2}\right) / \partial p_{2} \geq 0$. To see this, observe that:

$\partial \pi_{1}\left(p_{1}, p_{2}\right) / \partial p_{2}=c\left[p_{1}-\theta_{1}^{\prime}-\left(g_{1}-\theta_{1}\right) F_{1}\left(X_{1} / N_{1}\right)\right]$, and from (A5),

$X_{1}=a_{1}-b_{1} p_{1}+c p_{2}=b_{1}\left[p_{1}-\theta_{1}^{\prime}-\left(g_{1}-\theta_{1}\right) F_{1}\left(X_{1} / N_{1}\right)\right] \geq 0$.

\section{Proof of Proposition 5(b):}

If parameters are symmetric, $X_{1}^{C}=X_{2}^{C}=X^{C}, X_{1}^{B}=X_{2}^{B}=X^{B}$. By (A1), $X^{C}$ satisfies:

$\alpha-(2 \beta+\gamma) X^{C}-\theta^{\prime}-(g-\theta) F\left(X^{C} / N\right)=0$. By (A7), $X^{B}$ satisfies:

$\alpha-\left(2 \beta+r^{2} / \beta\right) X^{B}-\theta^{\prime}-(g-\theta) F\left(X^{C} / N\right)=0$.

By assumption $\beta_{1} \beta_{2}=\beta^{2}>r^{2}, \beta>\gamma$. Thus $\gamma>r^{2} / \beta$.

Comparing the above two equations, $X^{C}<X^{B}$. Utilizing the price function 2(a) or 2(b), $p^{C}=\alpha-(\beta+\gamma) X^{C}>\alpha-(\beta+\gamma) X^{B}=p^{B}$.

To compare expected profit, consider the profit function (3) at $X_{1}=X_{2}$. That is, $\pi(X)=(\alpha-$ $(\beta+\gamma) X) X+E\left(\pi_{s}\right)$, which is concave in $X$. From FOC, its optimal solution $X^{\#}$ satisfies: $\alpha-(2 \beta+$ 
$2 r) X^{\#}-\theta^{\prime}-(g-\theta) F\left(X^{\#} / N\right)=0$. Thus $X^{\#}<X^{C}<X^{B}$. Note the Cournot expected profit is $\pi\left(X^{C}\right)$, and the Bertrand expected profit is $\pi\left(X^{B}\right)$, thus $\pi\left(X^{\#}\right)>\pi\left(X^{C}\right)>\pi\left(X^{B}\right)$.

\section{Proof of Proposition 6:}

(a) The FOC of the cooperative and symmetric game is: $\alpha-(2 \beta+2 \gamma) X^{0}-\theta^{\prime}-(g-\theta) F\left(\frac{X^{0}}{N}\right) \equiv L^{0}$.

The FOC of the Cournot game is: $\alpha-(2 \beta+\gamma) X^{*}-\theta^{\prime}-(g-\theta) F\left(\frac{X^{*}}{N}\right) \equiv L^{*}$.

Comparing $L^{0}=0$ with $L^{*}=0$, it is easy to see that $X^{o}<X^{*}$.

(b) $\frac{\partial\left(X^{*}-X^{o}\right)}{\partial v}=\frac{\partial X^{*}}{\partial v}-\frac{\partial X^{o}}{\partial v}=-\frac{\frac{\partial L^{*}}{\partial v}}{\frac{\partial L^{*}}{\partial X}}-\left(-\frac{\frac{\partial L^{o}}{\partial v}}{\frac{\partial L^{o}}{\partial X}}\right)$, where,

$\frac{\partial L^{*}}{\partial v}=\frac{\partial L^{o}}{\partial v}=\frac{\theta}{v^{2}}\left(v\left(1-F(v)+\int_{v}^{B}(1-F(x) d x)>0\right.\right.$,

$\frac{\partial L^{*}}{\partial X}=-\frac{1}{(2 \beta+\gamma)+(g-\theta) / N f\left(\frac{X^{*}}{N}\right)}$, and,

$\frac{\partial L^{o}}{\partial X}=-\frac{1}{(2 \beta+2 \gamma)+(g-\theta) / N f\left(\frac{X^{o}}{N}\right)}$.

If $f^{\prime}(x) \leq 0$, and $f\left(X^{*} / N\right) \leq f\left(X^{0} / N\right)$, then $\frac{\partial\left(X^{*}-X^{o}\right)}{\partial v} \geq 0$.

For normal distribution, $\max \left|f\left(\frac{X^{o}}{N}\right)-f\left(\frac{X^{*}}{N}\right)\right|=f(\mu)=1 /(\sqrt{2 \pi} \sigma)$.

If $\gamma \geq(g-\theta) /(N \sqrt{2 \pi} \sigma)$, then $\frac{\partial\left(X^{*}-X^{o}\right)}{\partial v} \geq 0$.

Similarly,

$$
\begin{aligned}
& \frac{\partial\left(X^{*}-X^{o}\right)}{\partial g}=-\left(\frac{F\left(\frac{X^{*}}{N}\right)}{(2 \beta+\gamma)+\frac{g-\theta}{N f\left(\frac{X^{*}}{N}\right)}}-\frac{F\left(\frac{X^{0}}{N}\right)}{(2 \beta+2 \gamma)+(g-\theta) / N f\left(\frac{X^{0}}{N}\right)}\right) \leq 0 \text {, when the condition holds. } \\
& \frac{\partial\left(X^{*}-X^{o}\right)}{\partial \gamma}=-\left(\frac{1}{(2 \beta+\gamma)+\frac{g-\theta}{N f\left(\frac{X^{*}}{N}\right)}}-\frac{2}{(2 \beta+2 \gamma)+\frac{g-\theta}{N f\left(\frac{X^{0}}{N}\right)}}\right) \\
& =-\left(\frac{1}{(2 \beta+\gamma)+\frac{g-\theta}{N f\left(\frac{X^{*}}{N}\right)}}-\frac{1}{(\beta+\gamma)+(g-\theta) /(2 N) f\left(\frac{X^{0}}{N}\right)}\right) \geq 0 \text {, when the condition holds. } \\
& \frac{\partial\left(X^{*}-X^{o}\right)}{\partial \alpha}=\left(\frac{F\left(\frac{X^{*}}{N}\right)}{(2 \beta+\gamma)+\frac{g-\theta}{N f\left(\frac{X^{*}}{N}\right)}}-\frac{F\left(\frac{X^{0}}{N}\right)}{(2 \beta+2 \gamma)+(g-\theta) / N f\left(\frac{X^{0}}{N}\right)}\right) \geq 0 \text {, when the condition holds. }
\end{aligned}
$$

\section{Proof of Proposition 7:}

For network $i, \frac{\partial \pi_{i}}{\partial X_{k i}}=\alpha_{k i}-2 K \beta_{i} X_{k i}-K \gamma X_{k j}-\left(g_{i}-\theta_{i}\right) F_{i}\left(\sum_{k-1}^{K} \frac{X_{k i}}{N_{i}}\right)-\theta_{i}^{\prime}=0, k=1, \ldots, K$.

Adding the $K$ FOCs, we get:

$\sum_{k=1}^{K} \alpha_{k i}-2 K \beta_{i} \sum_{k=1}^{K} X_{k i}-K \gamma \sum_{k=1}^{K} X_{k j}-K \theta_{i}^{\prime}-K\left(g_{i}-\theta_{i}\right) F_{i}\left(\sum_{k-1}^{K} X_{k i} / N_{i}\right)=0, i=1,2$.

Comparing the above with FOC of aggregate planning, $\alpha_{i}-\left(2 \beta_{i}+\gamma\right) X_{i}-\theta_{i}^{\prime}-\left(g_{i}-\theta_{i}\right) F_{i}\left(\frac{X_{i}}{N_{i}}\right)=0$, $i=1$, 2, we get $X^{*=}=\sum_{k=1}^{K} X_{k i}^{*}$.

\section{Proof of Proposition 8:}

(a) Consider the additive demand function, $p_{s i}=\varepsilon_{i}\left(\theta_{c i}-\eta_{i} C_{i}\right),\left(\eta_{i}>0\right)$, first. Here, 
Therefore,

$$
p_{s i}=\left\{\begin{array}{c}
\theta_{c i} \varepsilon_{i}-\eta_{i} \varepsilon_{i}\left(N_{i}-\frac{X_{i}}{v_{i}}\right), \text { when } \varepsilon_{i} \geq v_{i}, \\
\theta_{c i} \varepsilon_{i}-\eta_{i} \varepsilon_{i}\left(N_{i}-\frac{X_{i}}{\varepsilon_{i}}\right), \text { when } \frac{X_{i}}{N_{i}} \leq \varepsilon_{i} \leq v_{i} .
\end{array}\right.
$$

$\frac{\partial \pi_{i}}{\partial X_{i}}=\alpha_{i}-2 \beta_{i} X_{i}-\gamma X_{j}-\theta_{c i}^{\prime}-\left(g_{i}-\theta_{c i}\right) F_{i}\left(\frac{X_{i}}{N_{i}}\right)+\int_{v_{i}}^{B_{i}} \frac{2 \eta_{i}\left(N_{i}-\frac{X_{i}}{v_{i}}\right) \varepsilon_{i}}{v_{i}} f_{i}\left(\varepsilon_{i}\right) d \varepsilon_{i}+\int_{X_{i} / N_{i}}^{v_{i}} 2 \eta_{i}\left(N_{i}-\frac{X_{i}}{\varepsilon_{i}}\right) \varepsilon_{i} f_{i}\left(\varepsilon_{i}\right) d \varepsilon_{i}$,

$\frac{\partial^{2} \pi_{i}}{\partial X_{i}^{2}}=-2 \beta_{i}-\left[\frac{\left(g_{i}-\theta_{i}\right)}{N_{i}}\right] f_{i}\left(\frac{X_{i}}{N_{i}}\right)-\int_{v_{i}}^{B_{i}} \frac{2 \eta_{i} \varepsilon_{i} f_{i}\left(\varepsilon_{i}\right)}{v_{i}^{2}} d \varepsilon_{i}-\int_{X_{i} / N_{i}}^{v_{i}} 2 \eta_{i} \varepsilon_{i} f_{i}\left(\varepsilon_{i}\right) d \varepsilon_{i}<0$,

$\frac{\partial^{2} \pi_{i}}{\partial X_{i} X_{j}}=-\gamma$.

Consider the multiplicative demand function, $p_{s i}=\theta_{c i} \varepsilon_{i} C_{i}^{-\eta_{i}}\left(\eta_{i}<1, \theta_{c i} \geq 0\right)$, next. Here,

Therefore,

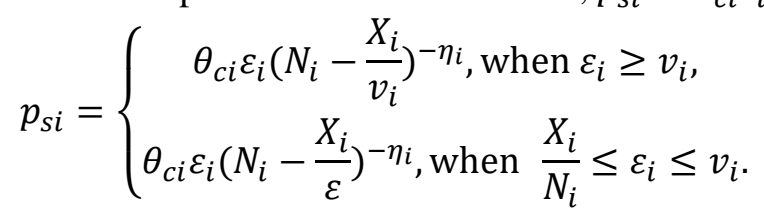

$$
\begin{aligned}
& \frac{\partial \pi_{i}}{\partial X_{i}}=\alpha_{i}-2 \beta_{i} X_{i}-\gamma X_{j}-g_{i} F_{i}\left(\frac{X_{i}}{N_{i}}\right)-\int_{v_{i}}^{B_{i}} \frac{\left(1-\eta_{i}\right)}{v_{i}}\left(N_{i}-\frac{X_{i}}{v_{i}}\right)^{-\eta_{i}} \theta_{c i} \varepsilon_{i} f_{i}\left(\varepsilon_{i}\right) d \varepsilon_{i} \\
&-\int_{X_{i} / N_{i}}^{v_{i}}\left(1-\eta_{i}\right)\left(N_{i}-\frac{X_{i}}{\varepsilon_{i}}\right)^{-\eta_{i}} \theta_{c i} f_{i}\left(\varepsilon_{i}\right) d \varepsilon_{i}, \\
& \begin{aligned}
\frac{\partial^{2} \pi_{i}}{\partial X_{i}^{2}}=-2 \beta_{i}-\left(\frac{g_{i}}{N_{i}}\right) f_{i}\left(\frac{X_{i}}{N_{i}}\right)-\int_{v_{i}}^{B_{i}} \frac{\eta_{i}\left(1-\eta_{i}\right)}{v_{i}^{2}}\left(N_{i}-\frac{X_{i}}{v_{i}}\right)^{-\eta_{i}-1} \theta_{c i} \varepsilon_{i} f_{i}\left(\varepsilon_{i}\right) d \varepsilon_{i} \\
-\int_{X_{i} / N_{i}}^{v_{i}} \frac{\eta_{i}\left(1-\eta_{i}\right)}{\varepsilon_{i}}\left(N_{i}-\frac{X_{i}}{\varepsilon_{i}}\right)^{-\eta_{i}-1} \theta_{c i} f_{i}\left(\varepsilon_{i}\right) d \varepsilon_{i},
\end{aligned}
\end{aligned}
$$

$\frac{\partial^{2} \pi_{i}}{\partial X_{i} X_{j}}=-\gamma$

The remainder of the proof is similar to that of Proposition 1.

(b) The proof is similar to the proof of Proposition 2. It is easy to verify that sign of $\left(\partial X_{i} / \partial \eta_{i}\right)=\operatorname{sign}$ of $\left(\partial^{2} \pi_{i} / \partial X_{i} \partial \eta_{i}\right) \geq 0$.

\section{Proof of Proposition 9:}

With uniform distribution, equation (14) becomes:

$\frac{\partial \pi_{i}}{\partial X_{i}}=\alpha_{i}-2 \beta_{i} X_{i}-\gamma \int_{0}^{d} X_{j}\left(l_{j}\right) d F_{l}\left(l_{j}\right)-\theta_{i}-\frac{\theta_{i}}{v_{i}} \frac{\left(\mu+l_{i}-v_{i}\right)^{2}}{4 l_{i}}-\left(g_{i}-\theta_{i}\right)\left(\frac{\frac{X_{i}}{N_{i}}-\left(\mu-l_{i}\right)}{2 l_{i}}\right)$.

Thus, $X_{i}\left(l_{i}\right)=\frac{\alpha_{i}-\gamma \int_{0}^{d} X_{j}\left(l_{j}\right) d F_{l}\left(l_{j}\right)-\theta_{i}-\frac{\theta_{i}\left(\mu+l_{i}-v_{i}\right)^{2}}{4 v_{i} l_{i}}+\frac{\left(g_{i}-\theta_{i}\right)\left(\mu-l_{i}\right)}{2 l_{i}}}{2 \beta_{i}+\frac{\left(g_{i}-\theta_{i}\right)}{2 N_{i} l_{i}}}$.

Let $\int_{0}^{d} X_{i}\left(l_{i}\right) d F_{l}\left(l_{i}\right)=W_{i}$. Taking the expectations of both sides of (A12) and utilizing the symmetry of parameters of the two networks, we get,

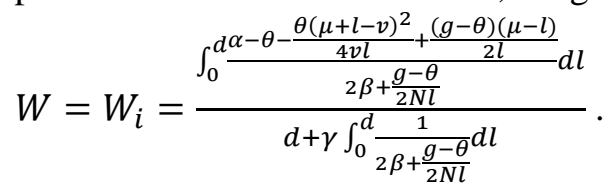


Plugging (A13) into (A12), we have

$$
X_{i}\left(l_{i}\right)=\frac{\alpha-\gamma W-\theta-\frac{\theta\left(\mu+l_{i}-v\right)^{2}}{4 v l}+\frac{(g-\theta)\left(\mu-l_{i}\right)}{2 l_{i}}}{2 \beta+\frac{(g-\theta)}{2 N l_{i}}} .
$$

Therefore, sign of $\left(\frac{\partial X_{i}}{\partial d}\right)=-\operatorname{sign}$ of $\left(\frac{\partial W}{\partial d}\right)$.

Taking derivatives of (A13) w.r.t. $d$, sign of $\left(\frac{\partial W}{\partial d}\right)$

$=\operatorname{sign}$ of $\left\{\frac{\alpha-\theta-\frac{\theta(\mu+d-v)^{2}}{4 v d d}+\frac{(g-\theta)(\mu-d)}{2 d}}{2 \beta+\frac{g-\theta}{2 N d}} d\left(1+\frac{\gamma}{d} \int_{0}^{d} \frac{1}{2 \beta+\frac{g-\theta}{2 N l}} d l\right)-\left(1+\frac{r}{2 \beta+\frac{g-\theta}{2 N d}}\right) \int_{0}^{d} \frac{\alpha-\theta-\frac{\theta(\mu+l-v)^{2}}{4 v l}+\frac{(g-\theta)(\mu-l)}{2 l}}{2 \beta+\frac{g-\theta}{2 N l}} d l\right\}$.

Since $\frac{1}{2 \beta+\frac{g-\theta}{2 N l}}$ increases in $l, \int_{0}^{d} \frac{1}{2 \beta+\frac{g-\theta}{2 N l}} d l<\frac{d}{2 \beta+\frac{g-\theta}{2 N d}}$, so $1+\frac{\gamma}{d} \int_{0}^{d} \frac{1}{2 \beta+\frac{g-\theta}{2 N l}} d l<1+\frac{r}{2 \beta+\frac{g-\theta}{2 N d}}$.

If $\frac{\alpha-\theta-\frac{\theta(\mu+l-v)^{2}}{4 v d}+\frac{(g-\theta)(\mu-l)}{2 l}}{2 \beta+\frac{g-\theta}{2 N l}}$ decreases in $l$, then:

$\frac{\alpha-\theta-\frac{\theta(\mu+d-v)^{2}}{4 v d}+\frac{(g-\theta)(\mu-d)}{2 d}}{2 \beta+\frac{g-\theta}{2 N d}} d<\int_{0}^{d} \frac{\alpha-\theta-\frac{\theta(\mu+l-v)^{2}}{4 v l}+\frac{(g-\theta)(\mu-l)}{2 l}}{2 \beta+\frac{g-\theta}{2 N l}} d l$, which will make sign of $\left(\frac{\partial W}{\partial d}\right)$ negative.

To find a sufficient condition for $\frac{\alpha-\theta-\frac{\theta(\mu+l-v)^{2}}{4 v d}+\frac{(g-\theta)(\mu-l)}{2 l}}{2 \beta+\frac{g-\theta}{2 N l}}$ to decreases in $l$, note that

$\frac{\alpha-\theta-\frac{\theta(\mu+l-v)^{2}}{4 v l}+\frac{(g-\theta)(\mu-l)}{2 l}}{2 \beta+\frac{g-\theta}{2 N l}}=\frac{2 N l(\alpha-\theta)-\frac{\theta N(\mu+l-v)^{2}}{2 v}+(g-\theta) N(\mu-l)}{4 \beta N l+g-\theta}$.

Taking its derivative w.r.t. $l$,

sign of $\partial\left(\frac{2 N l(\alpha-\theta)-\frac{\theta N(\mu+l-v)^{2}}{2 v}+(g-\theta) N(\mu-l)}{4 \beta N l+g-\theta}\right) / \partial l$

$=\operatorname{sign}$ of $\left[\frac{2 \beta \theta N^{2}}{v}(\mu+l-v)(\mu-l-v)+N(g-\theta)\left(2 \alpha-g-\frac{\theta}{v}(\mu+l)-4 \beta N \mu\right)\right]$.

Note that when $v \geq \mu$, $(\mu+l-v)(\mu-l-v)<0$.

Therefore, if $2 \alpha-g-\frac{\theta}{v}(\mu+l)-4 \beta N \mu \leq 0$, then $\frac{\partial W}{\partial d} \leq 0$; and hence, by (A14), $\frac{\partial X_{i}}{\partial d} \geq 0$.

\section{$\underline{\text { References }}$}

Cachon G.P., S. Netessine. 2004. Game Theory in Supply Chain Analysis. In book: Handbook of quantitative supply chain analysis: modeling in the e-business era, Publisher: Kluwer, Editors: SimchiLevi, David, David Wu, Max Shen, pp.13 - 59.

Cheng L. (1985), Comparing Bertrand and Cournot Equilibria: A Geometric Approach. Rand Journal of Economics 16(1), 146-151.

Moulin, H. (1986). Game Theory for the Social Science. New York University Express. 